\title{
Kilohertz gravitational waves from binary neutron star remnants: Time-domain model and constraints on extreme matter
}

\author{
Matteo Breschiø, ${ }^{1}$ Sebastiano Bernuzzi, ${ }^{1}$ Francesco Zappa $\odot,{ }^{1}$ Michalis Agathos, ${ }^{1}$ \\ Albino Perego, ${ }^{2,3}$ David Radice, ${ }^{4,5,6,7}$ and Alessandro Nagar ${ }^{8,9,10}$ \\ ${ }^{1}$ Theoretisch-Physikalisches Institut, Friedrich-Schiller-Universität Jena, 07743, Jena, Germany \\ ${ }^{2}$ Dipartimento di Fisica, Universitá di Trento, Via Sommarive 14, 38123 Trento, Italy \\ ${ }^{3}$ Istituto Nazionale di Fisica Nucleare, Sezione di Milano-Bicocca, \\ Piazza della Scienza 20100, Milano, Italy \\ ${ }^{4}$ Department of Physics, The Pennsylvania State University, University Park, Pennsylvania 16802, USA \\ ${ }^{5}$ Department of Astronomy \& Astrophysics, The Pennsylvania State University, \\ University Park, Pennsylvania 16802, USA \\ ${ }^{6}$ Institute for Advanced Study, 1 Einstein Drive, Princeton, New Jersey 08540, USA \\ ${ }^{7}$ Department of Astrophysical Sciences, Princeton University, 4 Ivy Lane, \\ Princeton, New Jersey 08544, USA \\ ${ }^{8}$ Centro Fermi-Museo Storico della Fisica e Centro Studi e Ricerche Enrico Fermi, 00184 Roma, Italy \\ ${ }^{9}$ Istituto Nazionale di Fisica Nucleare, Sezione di Torino, Via P. Giuria 1, 10125 Torino, Italy \\ ${ }^{10}$ Institut des Hautes Etudes Scientifiques, 91440 Bures-sur-Yvette, France
}

(Received 6 September 2019; published 14 November 2019)

\begin{abstract}
The remnant star of a neutron star merger is an anticipated loud source of kilohertz gravitational waves that conveys unique information on the equation of state of hot matter at extreme densities. Observations of such signals are hampered by the photon shot noise of ground-based interferometers and pose a challenge for gravitational-wave astronomy. We develop an analytical time-domain waveform model for postmerger signals informed by numerical relativity simulations. The model completes effective-one-body waveforms for quasicircular nonspinning binaries in the kilohertz regime. We show that a template-based analysis can detect postmerger signals with a minimal signal-to-noise ratio (SNR) of 8.5, corresponding to GW170817like events for third-generation interferometers. Using Bayesian model selection and the complete inspiralmerger-postmerger waveform model it is possible to infer whether the merger outcome is a prompt collapse to a black hole or a remnant star. In the latter case, the radius of the maximum mass (most compact) nonrotating neutron star can be determined to kilometer precision. We demonstrate the feasibility of inferring the stiffness of the equation of state at extreme densities using the quasiuniversal relations deduced from numerical-relativity simulations.
\end{abstract}

DOI: 10.1103/PhysRevD.100.104029

\section{INTRODUCTION}

The gravitational-wave (GW) signal GW170817 is compatible with the inspiral of a binary neutron star (BNS) of chirp mass $\mathcal{M} \sim 1.186(1) M_{\odot}$, mass ratio $q \sim[1,1.34]$ and tidal deformability parameter distributed around $\tilde{\Lambda} \sim 300$ and smaller than $\sim 800$ [1-3]. The merger frequency of a BNS GW can be accurately predicted using numerical relativity (NR) results [4]. From the probability distribution of $\tilde{\Lambda}$ measured for GW170817 one finds that the merger frequency falls in the broad range $f_{\mathrm{mrg}} \sim(1.2,2) \mathrm{kHz}$ (Fig. 1). The sensitivity of the detectors in August 2017 was insufficient to clearly identify a signal at frequencies $f \gtrsim f_{\mathrm{mrg}}$ [5,6]. Indeed, LIGO-Virgo searches for short $(\lesssim 1 \mathrm{~s})$, intermediate $(\lesssim 500 \mathrm{~s})$ and long (days) postmerger transients from a neutron star (NS) remnant resulted in upper limits of more than one order of magnitude larger than those predicted by basic models of quasiperiodic sources [7-12]. Various works have suggested that for GW170817-like sources postmerger frequencies are accessible only by improving the design sensitivity of current detectors of a factor $2-3$ or with next-generation detectors [5,13-15].

NR simulations predict that BNS mergers can form a black hole $(\mathrm{BH})$ from gravitational collapse of the merged object or a NS remnant depending on the binary mass and the NS matter equation of state (EOS), e.g., [17-22]. NS remnants can collapse on dynamical $[\sim \mathcal{O}(10) \mathrm{ms}$, shortlived remnant] or longer timescales (long-lived remnant), but can also reach a stable NS configuration. Kilohertz GWs contain the imprint of the merger remnant dynamics. The main signature is a short GW transient peaking at a few characteristic frequencies, the dominant one being associated with twice the rotation frequency of the remnant NS at 


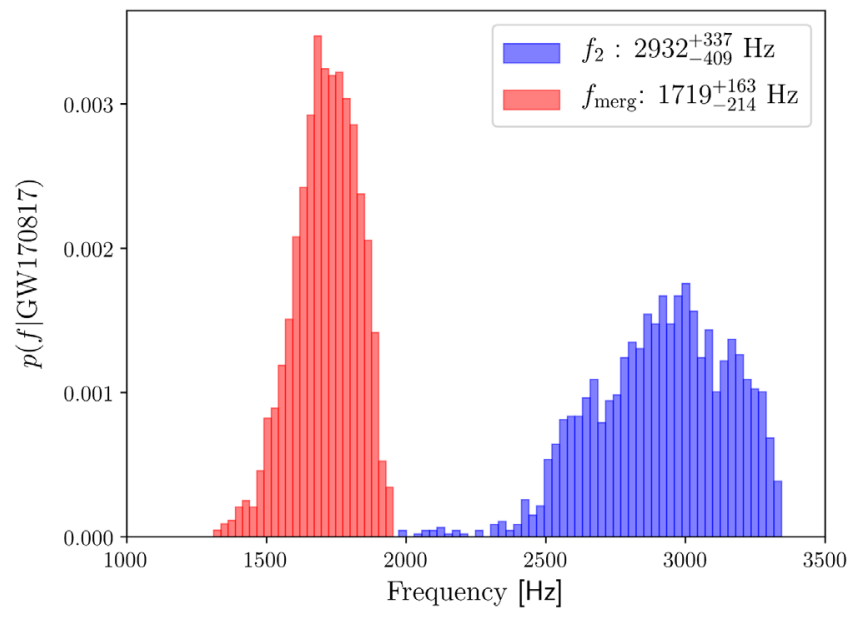

FIG. 1. Gravitational-wave merger $f_{\mathrm{mrg}}$ and postmerger peak $f_{2}$ frequency for GW170817. The distributions are estimated from the LIGO-Virgo posterior distributions [3] for the $\tilde{\Lambda}$ parameters using (i) the quasiuniversal relation proposed in [4] for the merger frequency; (ii) the relation proposed in [16] and further refined in this work for the postmerger peak frequency. The distribution of $f_{2}$ is cut at $\kappa_{2}^{\mathrm{T}}<70$ to exclude binaries that undergo prompt collapse at merger.

$f_{2}>f_{\mathrm{mrg}}[16,21-30]$. The transient is more luminous for a short-lived remnant than for a long-lived one; an absolute upper limit to the energy per unit mass is $\lesssim 0.126\left(\frac{M}{2.8 M_{\odot}}\right) M_{\odot} \mathrm{c}^{2}$, where $M$ is the binary mass [12]. Long postmerger transients are also possible for NS remnants developing nonaxisymmetric instabilities and/or magnetars, but they are expected to be less luminous than the GWs on dynamical timescales, e.g., [7-11]. Recent analysis of GW170817 based on premerger GWs combined with the pulsar constraints on the maximum mass largely disfavor prompt collapse to $\mathrm{BH}$ [31]. Using the NR relation between the frequency $f_{2}$ and the tidal deformability derived in [16] and the LIGO-Virgo posteriors for GW170817, one finds that a tentative wave with peak luminosity larger than $0.1 \times 10^{56} \mathrm{erg} \cdot \mathrm{s}^{-1}$ could have been detected at $f_{2} \sim[2.5,3.2] \mathrm{kHz}$ (Fig. 1) if the instruments were more sensitive. This is compatible with the interpretation of the electromagnetic counterparts that suggests the formation of a short-lived NS remnant [32-36], although other scenarios are possible [37-41].

The data analysis of (short duration) postmerger signals can be performed with either morphology-independent approaches $[14,42]$ or using matched filtering techniques based on waveform templates. While matched filtering is proven to be an optimal method in the case of Gaussian noise [43], its performance for postmerger analysis remains unclear due to the uncertainties of postmerger templates. Current postmerger models comprise frequency-domain statistical representation of NR waveforms $[13,44]$ or simple analytical models [27,45-47]. A common aspect of all these approaches is the use of NR information in terms of quasiuniversal (EOS-independent) relations for the characteristic frequencies $[16,25,28,48-51]$. The relevance of these relations is twofold: on one hand they are used for waveform modeling, on the other hand they can be used to extract information from the analysis.

Observations of kilohertz GWs from NS remnants can deliver constraints on the EOS of matter in a regime at which nuclear interactions are still very uncertain. For a canonical binary of mass $M=(1.4+1.4) M_{\odot}$, tidal interactions in the inspiral-merger part of the GW signal mostly inform about the EOS at about twice the nuclear saturation density $\rho_{0} \simeq 2.3 \times 10^{14} \mathrm{~g} \mathrm{~cm}^{-3}$, corresponding to the maximal densities of the binary components [31,52]. However, NS remnants formed in mergers reach densities $\sim 3-5 \rho_{0}$ and temperatures in excess of $\sim 50 \mathrm{MeV}$, e.g., [53]. The strongest constraints on the EOS at those extreme densities are currently provided by the mass measurements of two pulsars in binary systems [54,55]. The latter give lower bounds for the maximum mass of nonrotating stable NS in equilibrium ( $M_{\max }^{\mathrm{TOV}}$, hereafter simply referred to as the maximum NS mass): $M_{\max }^{\mathrm{TOV}} \gtrsim(2.01 \pm 0.04) M_{\odot} \quad(\mathrm{PSR}$ $\mathrm{J} 0348+0432)$ [54] and $M_{\max }^{\mathrm{TOV}} \gtrsim(2.17 \pm 0.11) M_{\odot}$ (PSR $\mathrm{J} 0740+6620)[55]$.

Additional constraints on matter at extreme densities can be inferred from the kilohertz GW from merger remnants by extracting NS properties via quasiuniversal relations $[16,48,56]$. Moreover, new degrees of freedom or matter phases at $\sim 3-5 \rho_{0}$ can impact the remnant dynamics and leave detectable imprints on the GW. Case studies considered matter models including hyperon production $[57,58]$ or zero-temperature models of phase transitions to quarkdeconfined matter $[59,60]$. The detectability of these effects crucially depends on the densities at which the EOS softening (or stiffening) takes place and would in principle need detailed waveform models that are presently not available.

In this paper we construct the first phase-coherent inspiral-merger-postmerger model for the BNS GW spectrum and demonstrate its applications to constrain the NS EOS in GW astronomy observations.

Section II introduces a NR postmerger model for quasicircular binaries called NRPM, based on the quasiuniversal relations of [16] and implemented using the NR database of the Computational Relativity (CoRe) Collaboration [61].

Section III discusses performances of NRPM using a validation set of NR simulations. Section IV discusses how to complete effective-one-body waveforms with NRPM in order to obtain a phase-coherent model of the complete inspiral-merger-postmerger waveform, valid from the circular adiabatic regime to the kilohertz regime.

Section V demonstrates the use of the model in templatebased Bayesian data analysis applications. We discuss the minimal requirement for postmerger detection. We demonstrate how to infer prompt collapse using our complete 
spectrum model and Bayesian model selection. We show how to set constraints on the minimum NS radius from a single event. Finally, we discuss how to infer EOS stiffness at the extreme densities reached in the merger remnant.

Conventions For waveform modeling we mostly use geometric units $c=G=1$ and measure masses in terms of solar masses $M_{\odot}$. The waveform strain is decomposed in multipoles as

$$
h_{+}-\mathrm{i} h_{\times}=D_{L}^{-1} \sum_{\ell=2}^{\infty} \sum_{m=-\ell}^{\ell} h_{\ell m}(t)_{-2} Y_{\ell m}(l, \psi),
$$

where $D_{L}$ is the luminosity distance and ${ }_{-2} Y_{\ell m}$ are the $s=-2$ spin-weighted spherical harmonics. In this paper we shall compute the strain from the equation above assuming only the $\ell=2, m= \pm 2$ modes and symmetry across the orbital plane. ${ }^{1}$ The $\ell=m=2$ waveform mode is decomposed in amplitude $A(t)$ and phase $\phi(t)$ as

$$
h_{22}(t)=A(t) \exp (-\mathrm{i} \phi(t)) ; \quad \omega(t)=\dot{\phi}(t),
$$

where $\omega(t)$ also indicates the GW frequency and the dot denotes the time derivative. The corresponding spherical harmonics are

$$
{ }_{-2} Y_{2, \pm 2}(l, \psi)=\sqrt{\frac{5}{64 \pi}}(1 \pm \cos (l))^{2} e^{ \pm 2 i \psi},
$$

so that one obtains

$$
\begin{aligned}
h_{+}-\mathrm{i} h_{\times} \approx & \sqrt{\frac{5}{4 \pi}} \frac{A(t)}{D_{L}}\left[\frac{1}{2}\left(\cos ^{2}(l)+1\right) \cos (\phi(t))\right. \\
& -\mathrm{i} \cos (l) \sin (\phi(t))]
\end{aligned}
$$

where one sets $\psi=0$. We work with quantities rescaled by the total binary mass, i.e.,

$$
\hat{\omega}:=M \omega=2 \pi \hat{f}, \quad \hat{t}:=t / M, \quad \hat{A}:=A / M,
$$

and further define the moment of merger $\left(\hat{t}_{\mathrm{mrg}}=0\right)$ as the time of the peak of $A(t)$ (Fig. 2). Note that the time $\hat{t}$ refers to the retarded time in the case of the NR data. The binary mass is indicated with $M=M_{A}+M_{B}$, the mass ratio $q=M_{A} / M_{B} \geq 1$ and the symmetric mass ratio $\nu=M_{A} M_{B} / M^{2}$. GW spectra and frequencies are instead discussed and shown in SI units with distances expressed in Mpc.

\footnotetext{
${ }^{1}$ We are considering here only nonprecessing systems.
}

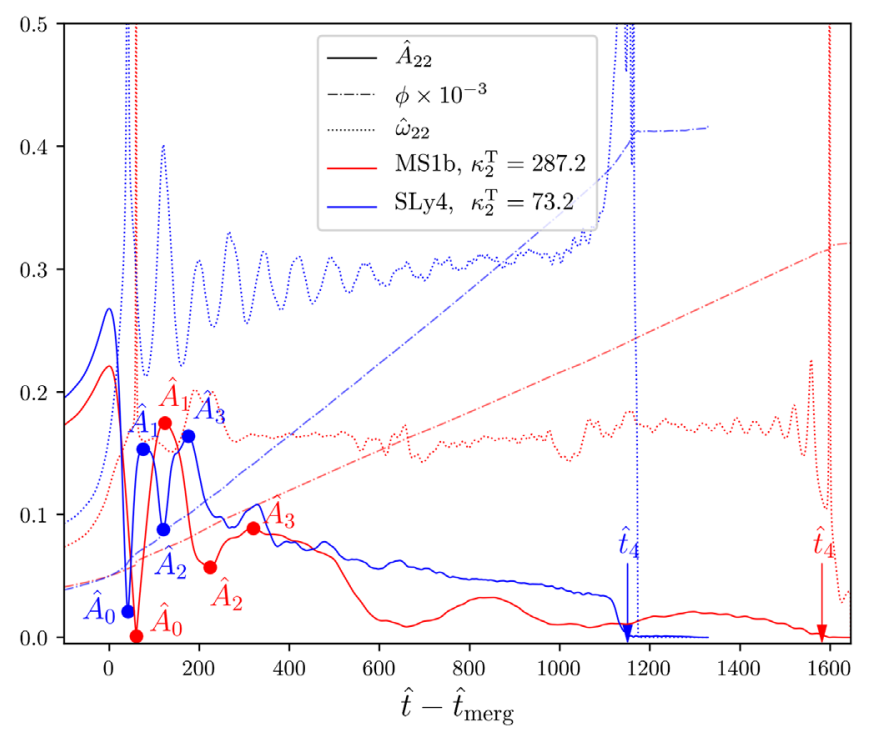

FIG. 2. Merger and postmerger waveform from two very different BNS with mass $M=(1.35+1.35) M_{\odot}$. The MS1b BNS is an example of a long-lived remnant, and the SLy BNS is an example of a short-lived remnant collapsing at $\hat{t} \sim 1200$ after merger time, $\hat{t}=\hat{t}_{\mathrm{mrg}}$. In both cases the postmerger waveform amplitude has characteristic maxima and minima $\hat{A}_{i}$ at times $\hat{t}_{i}$ with $i=0, \ldots, 3$. Note the jump in the phase at $\hat{t}_{0}$, where the instantaneous frequency is not defined.

\section{NRPM MODEL}

Our postmerger model builds on the results of [12,16,62] that showed the postmerger frequency peak correlates with the tidal polarizability parameter

$$
\kappa_{2}^{\mathrm{T}}=\frac{3}{2}\left[\Lambda_{2}^{\mathrm{A}}\left(X_{\mathrm{A}}\right)^{4} X_{\mathrm{B}}+\Lambda_{2}^{\mathrm{B}}\left(X_{\mathrm{B}}\right)^{4} X_{\mathrm{A}}\right],
$$

where $\Lambda_{2}^{i} \equiv 2 k_{2}^{i}\left(M_{i} / R_{i}\right)^{5} / 3$, with $i=(A, B)$, are the dimensionless quadrupolar tidal polarizability parameters of the individual stars $[63,64], k_{2}^{i}$ the dimensionless quadrupolar Love numbers [65-68], and $\left(M_{i}, R_{i}\right)$ the mass and radius and $X_{\mathrm{i}} \equiv M_{\mathrm{i}} / M$. Here we derive similar relations also for other characteristic frequencies of the spectrum and for the waveform's amplitudes and characteristic times. For nonspinning and slowly spinning BNS, each of those quantities can be approximately modeled in terms of the following set of physical parameters:

$$
\boldsymbol{\theta}=\left(\nu, M, \kappa_{2}^{\mathrm{T}}\right),
$$

that defines NRPM's parameter space. The latter choice is one of the key differences with respect to previous timedomain models $[27,45,46]$. Other important differences are the use of the largest-to-date set of NR simulations and the possibility of constructing a time-domain approximant that is phase coherent with inspiral-merger models (see Sec. IV). 
We use 148 simulations of the Computational Relativity (CoRe) Collaboration [61], plus 24 simulations in part reported in [69] and in part unpublished. The set of simulations covers the range $q \in[1,1.5]$ and $\kappa_{2}^{\mathrm{T}} \in[73,458]$.

Figure 2 illustrates some of the qualitative features common to all the merger + postmerger NR waveforms for short- and long-lived NS remnants. The waveform frequency at early times is approximately constant around the $\hat{f}_{2}$ value. In many waveforms a further frequency modulation is clearly present in the first milliseconds after merger. This feature is interpreted as the couplings between $\hat{f}_{2}$ and a radial pulsation mode $\hat{f}_{0}$, in analogy to what happens with nonlinear perturbations of equilibrium NS [24,70-72]. In the latter case, nonlinear couplings between proper modes result in new frequencies given by $\hat{f}_{2 \pm 0}=\hat{f}_{2} \pm \hat{f}_{0}$. In the case of BNS mergers, the two secondary peaks in the GW spectra can be interpreted as the nonlinear pulsations of the remnant $\hat{f}_{2 \pm 0}$ [24]. These secondary frequency peaks in the spectrum are well studied, e.g., $[21,22,73,74]$ and can be clearly seen in Fig. 5.

Although we will often refer to discrete frequencies (spectral peaks), we stress that the GW frequency is not constant but evolves (chirplike) as the remnant becomes more compact and eventually collapses (see SLy data in Fig. 2). The largest GW luminosity is emitted at early times after merger at which $\hat{f}(t)$ is approximated by a certain combination of $\hat{f}_{2}, \hat{f}_{2 \pm 0}$ [75]. The waveform's amplitude after the merger peak has typically a minimum, a maximum and at least a second oscillation. In Fig. 2 these extrema are labeled as $\hat{A}_{i}$ and occur at times $\hat{t}_{i}$ with $i=0,1,2,3$ where the minima have even indices. Note that at $\hat{t}_{0}$ the GW phase has a jump and the instantaneous frequency is not defined; this corresponds to a moment in which the remnant has a strongly suppressed quadrupolar deformation. At timescales $\sim 10-20 \mathrm{~ms}$ corresponding to $\hat{t} \sim 1000-2000$ $\left(M \sim 2.7 M_{\odot}\right)$ the remnant has either collapsed (shortlived) or dissipated most of its energy via GWs. There is no significant GW emission at timescales $\tau \gtrsim 100 \mathrm{~ms}[29,76]$ (see also the Appendix C).

In the following we describe in detail the construction of the time-domain model and how the NR information is extracted.

\section{A. Time-domain model}

\section{Frequency and phase}

We assume the GW frequency is composed of the three main characteristic frequencies $\hat{f}_{2-0}<\hat{f}_{2}<\hat{f}_{2+0}$ and construct a $\mathcal{C}^{1}$ model for $\hat{\omega}(t)$ as follows. The frequency model starts at $\hat{t}=\hat{t}_{\mathrm{mrg}}=0$ with the value of the merger frequency $\hat{\omega}_{\text {mrg }}$ and its derivative $\dot{\hat{\omega}}_{\text {mrg }}$ taken either from NR fits or from an inspiral-merger time-domain approximant (see Sec. IV). We impose

$$
\begin{gathered}
\hat{\omega}\left(\hat{t}_{\mathrm{mrg}}\right)=\hat{\omega}_{\mathrm{mrg}} \\
\hat{\omega}\left(\hat{t}_{0} \leq \hat{t} \leq \hat{t}_{1}\right)=\hat{\omega}_{2-0} \\
\hat{\omega}\left(\hat{t}_{2}\right)=\hat{\omega}_{2+0} \\
\hat{\omega}\left(\hat{t} \geq \hat{t}_{3}\right)=\hat{\omega}_{2},
\end{gathered}
$$

and use a cubic interpolant to join $\hat{\omega}_{\text {mrg }}$ to $\hat{\omega}_{2-0}$ in the interval $\left(\hat{t}_{\mathrm{mrg}}, \hat{t}_{0}\right)$ fixing the values of the function and of the first derivatives at the interval's extrema. The derivative at $\hat{t}_{0}$ is taken as $\dot{\hat{\omega}}\left(\hat{t}=\hat{t}_{0}\right)=0$. The frequency oscillation in the intervals $\left(\hat{t}_{1}, \hat{t}_{2}\right)$ and $\left(\hat{t}_{2}, \hat{t}_{3}\right)$ is modeled with a sine function in such a way that $\hat{\omega}_{2+0}$ is a maximum and preserving the continuity and the differentiability of $\hat{\omega}(t)$. Note the model can be reduced to a single-frequency one by simply joining $\hat{\omega}_{\mathrm{mrg}}$ to $\hat{\omega}_{2}$ at $\hat{t}_{3}$ and omitting $\hat{\omega}_{2 \pm 0}$. The phase of the waveform is finally given by integrating the frequency model,

$$
\phi(\hat{t})=\int_{0}^{\hat{t}} \hat{\omega}\left(\hat{t}^{\prime}\right) \mathrm{d} \hat{t}^{\prime}+\phi_{0}
$$

where $\phi_{0}$ is either arbitrarily chosen or fixed by requiring continuity with an inspiral-merger phase.

\section{Amplitude}

We assume the postmerger amplitude has two minima, $\hat{A}_{i}$ with $i=0,2$, and two maxima, $\hat{A}_{i}$ with $i=1,3$, and that it decays exponentially after the second maximum. A $\mathcal{C}^{1}$ model for $\hat{A}(t)$ is constructed assuming

$$
\begin{gathered}
\hat{A}\left(\hat{t}_{\mathrm{mrg}}\right)=\hat{A}_{\mathrm{mrg}} \\
\hat{A}\left(\hat{t}_{i}\right)=\hat{A}_{i} \\
\hat{A}\left(\hat{t} \geq \hat{t}_{3}+5\right)=\hat{A}_{3} \exp \left[-\alpha\left(\hat{t}-\hat{t}_{3}\right)\right],
\end{gathered}
$$

and using sine waves to connect maxima and minima. We define fractional amplitudes $\beta_{i}=\hat{A}_{i} / \hat{A}_{\mathrm{mrg}}$ with $i=0,1,2$, 3 of the extrema with respect to the merger amplitude. The damping term $\alpha$ is set as the timescale at which the waveform amplitude is $1 / 100$ of the merger value, i.e., when $\hat{A}$ falls below the threshold

$$
\beta_{4}=10^{-2} .
$$

Indicating $\hat{t}_{4}$ as such a time, one obtains

$$
\alpha=\frac{\ln \left(100 \beta_{3}\right)}{\hat{t}_{4}-\hat{t}_{3}} .
$$

The timescale $1 / \alpha$ is identified from simulations and has range $\sim(3,70) \mathrm{ms}$ for BNS masses distributed 
$M \sim(2.5,3) M_{\odot}$, if no collapse to a BH happens before [75] (see also Sec. II B 2 for discussion on BH collapse).

\section{B. NR information}

The model's parameters are summarized in Table I; their values are fixed by constructing interpolating formulas of NR data on the space of parameters $\boldsymbol{\theta}$.

\section{Frequencies, amplitudes and times}

The frequency information is extracted from the spectra by identifying the three dominant peak frequencies. Amplitudes $\hat{A}_{i}$ and the related times $\hat{t}_{i}$ are extracted from the waveforms (Fig. 2). Specifically, we construct fit models using the variable [77] (see also Appendix A)

$$
\xi=\kappa_{2}^{T}+c(1-4 \nu),
$$

where the constant $c$ is also a fitting parameter. The frequency and amplitude at merger $\hat{A}_{\text {mrg }}$ and the peak frequencies are well described by rational functions in the form

$$
F_{\text {Rational }}\left(\kappa_{2}^{\mathrm{T}}, q\right)=F_{0} \frac{1+n_{1} \xi+n_{2} \xi^{2}}{1+d_{1} \xi+d_{2} \xi^{2}}
$$

where $\left(F_{0}, n_{1}, n_{2}, d_{1}, d_{2}\right)$ are the fitting parameters. The amplitudes $\hat{A}_{i}$ for $i=0,1,2,3$ and the times $\hat{t}_{i}$ are instead fit by linear polynomials in $\xi$,

$$
F_{\text {Linear }}\left(\kappa_{2}^{\mathrm{T}}, q\right)=p_{0}+p_{1} \xi
$$

where $\left(p_{0}, p_{1}\right)$ are fitting parameters. The results of the fits are shown in Table I.

As an example, the peak frequency fits are shown in Fig. 3. The uncertainty of the NR data computed from simulations at multiple grid-resolutions is shown in the plot as bars, if available. Note that the $\hat{f}_{2}$ peak's determination is affected by a further error of $\sim 2-8 \%$ due to the discrete Fourier transform; larger errors affect the $\hat{f}_{2 \pm 0}$ determination. The $\chi^{2}$ coefficients for the frequencies fit are typically $\sim 10^{-4}$ (note that the merger frequency has $\chi^{2} \sim 10^{-5}$ ), but some outliers are visible from the plots at small $\xi$, or equivalently small $\kappa_{2}^{T}$ (since these points correspond to $q \sim 1$ ). We note that most of these data points correspond to low-resolution simulations for which error bars either cannot be computed (one resolution available) or are unreliable (two low resolutions available). For example, the ENG simulation at $\kappa_{2}^{\mathrm{T}} \sim 80$ is a high-mass $M=(1.7+1.7) M_{\odot}$ BNS simulated at a maximal grid resolution of $h \approx 0.365 \mathrm{~km}$ that does not guarantee convergence even for the inspiral-merger (cf. [78-80] and Appendix C). The frequency $\hat{f}_{2+0}$ model is the most uncertain for the available data.
Table I (see also Appendix A) shows that, while postmerger amplitude fits are well captured by the model $\left(\chi^{2} \sim 10^{-3}\right)$, the postmerger times are more uncertain $\left(\chi^{2}>1\right)$ with the uncertainty growing for larger times. This is expected since the quantities at later times are less correlated with premerger parameters and NR data are themselves more uncertain the longer the simulation is. While uncertainties on "late-time" quantities do not affect significantly the time-domain waveform (see discussion in Sec. III), they can affect the Bayesian parameter estimation (Sec. V). Notably, the damping parameter $\alpha$ is degenerate with part of the waveform amplitude in Fourier space, and therefore fit biases can affect the estimation of the luminosity distance.

\section{Prompt collapse}

NR simulations indicate that a NS binary merger will be followed by a prompt collapse to a $\mathrm{BH}$, if the total gravitational mass $M$ of the binary exceeds a threshold mass. The latter can be roughly estimated as $[19,20]$

$$
M_{\mathrm{thr}}=k_{\mathrm{thr}} M_{\mathrm{max}}^{\mathrm{TOV}},
$$

where $M_{\max }^{\mathrm{TOV}}$ is the gravitational mass of the heaviest stable nonrotating NS. Both $M_{\max }^{\mathrm{TOV}}$ and $k_{\mathrm{thr}}$ depend, in general, on the EOS, mass ratio, and spins. For a sample of hadronic EOS and equal-mass nonspinning binaries, the threshold parameter in Eq. (15) is found in the range $1.3 \lesssim k_{\text {thr }} \lesssim 1.7$ $[19,20,31]$. Moreover, $k_{\text {thr }}$ shows an approximately EOSindependent linear behavior in the compactness $C$ of a reference nonrotating NS at equilibrium; see [31] for a recent collection of literature data, fit recalibration and discussion. Despite several NR efforts, it remains challenging to construct a EOS-independent (universal) relation for $M_{\text {thr }}$ that is accurate and robust across the entire parameter space. A data analysis approach based on Eq. (15), NRPM and EOS inference is outlined in Appendix B.

We follow here an alternative route. By analyzing the NR data of the CoRe Collaboration, we have found that all the 30 prompt-collapse mergers are captured by the condition $\kappa_{2}^{\mathrm{T}}<80$; see also Ref. [12]. Further combining the estimate with Eq. (15) for a sample of nonrotating NS model with 13 EOS leads to the following criterion for prompt collapse [12]:

$$
\kappa_{2}^{\mathrm{T}}<\kappa_{\mathrm{thr}}^{\mathrm{T}}=80 \pm 40 .
$$

We adopt the above criterion in NRPM. In the context of a Bayesian analysis, the threshold value can be either prescribed or included in the set of intrinsic parameters.

This assumption is a simplification as the promptcollapse threshold is primarily determined by the EOS pressure support at large densities (or the maximum mass). For example, for a EOS sufficiently soft at the postmerger densities $\rho \gtrsim 3 \rho_{0}$, where $\rho_{0}$ is the nuclear density, but 


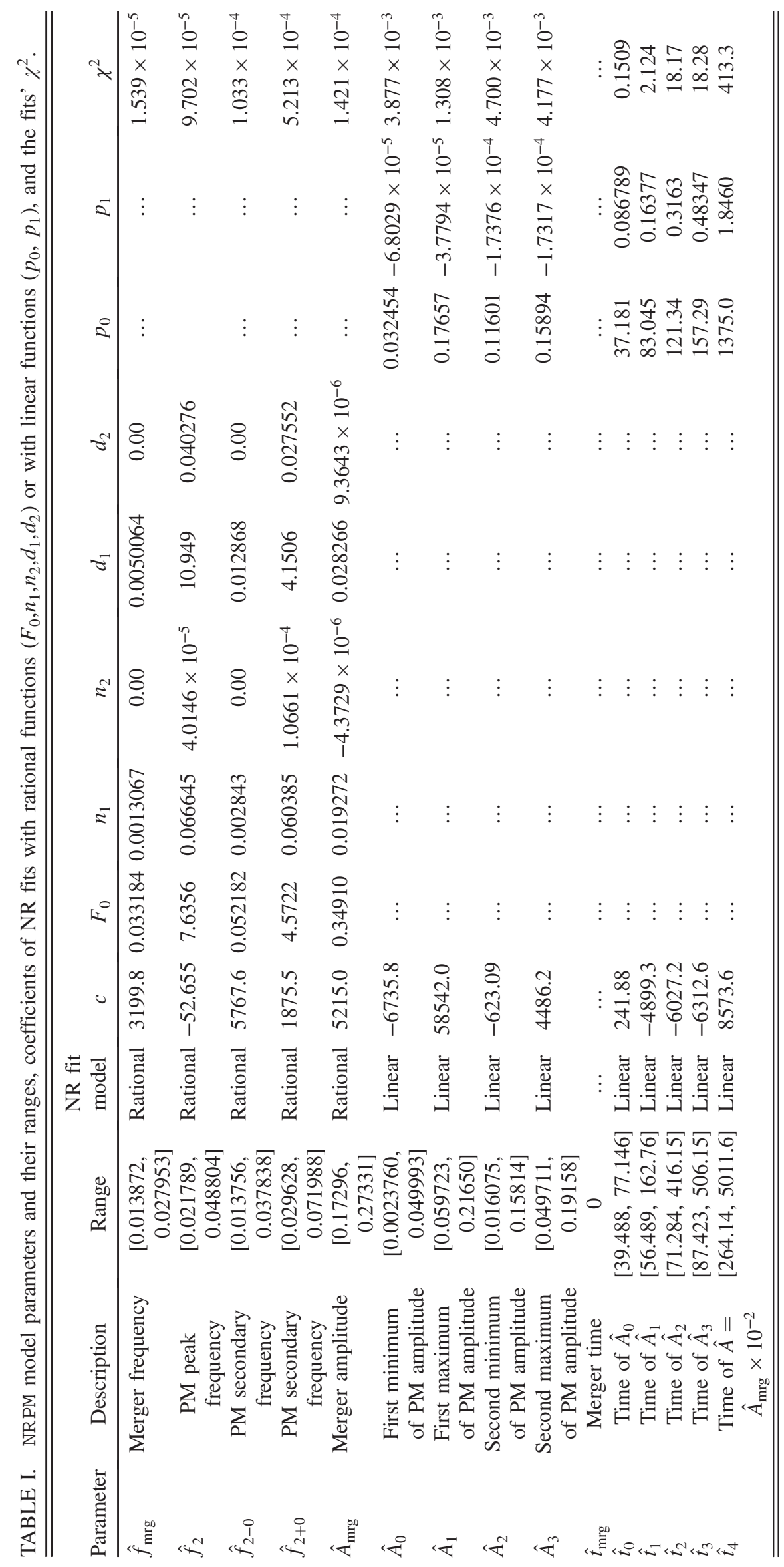




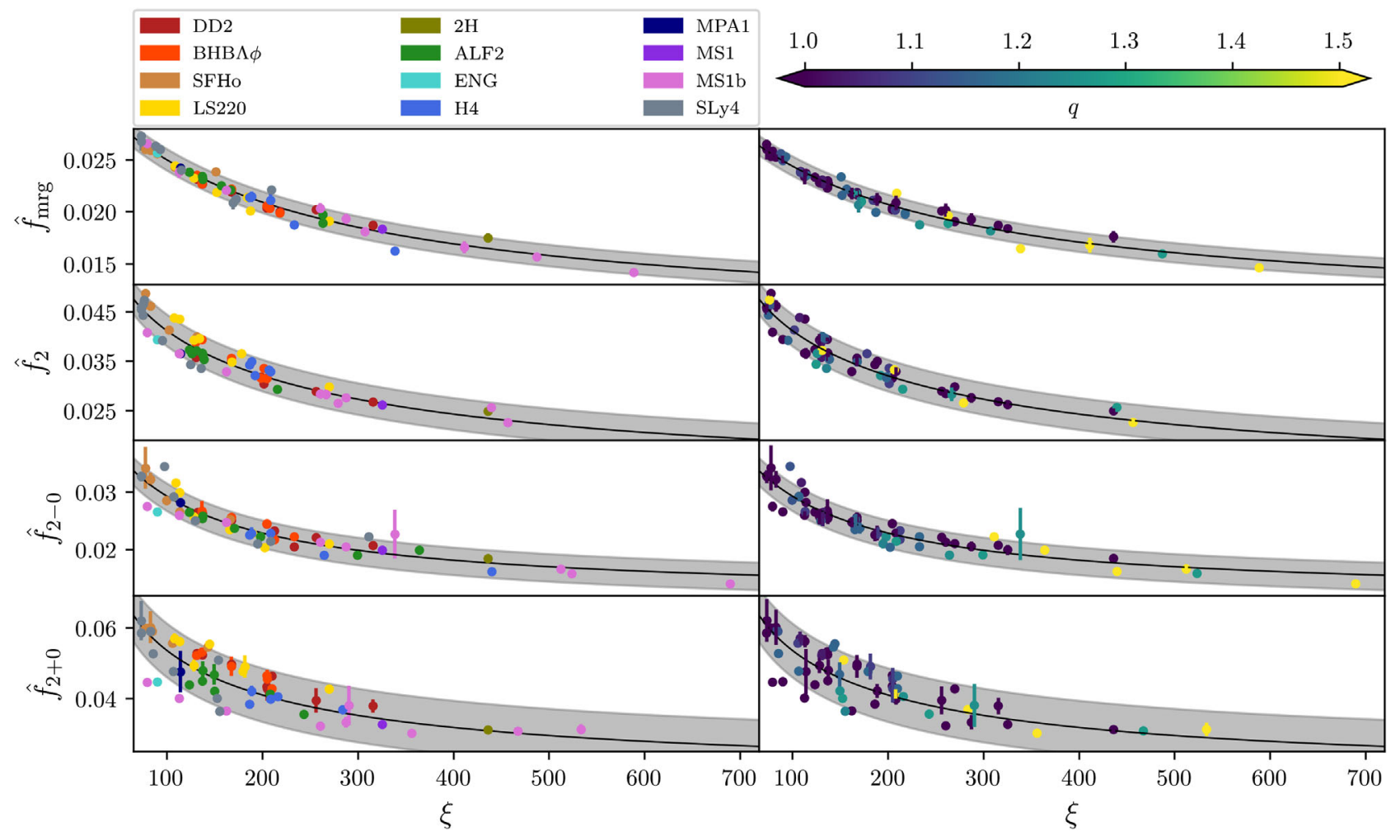

FIG. 3. Characteristic frequency information from NR simulations. Markers represent the frequencies extracted from the NR data and the uncertainties are estimated using simulations at different resolutions; the black lines are the fits and the gray bands are the $90 \%$ credible regions. Left and right panels show the same data: the colors on the left panel correspond to the EOS variation, those on the right panel to the mass ratio.

admitting small compactness at inspiral densities $\left(\rho \sim 2 \rho_{0}\right)$, Eq. (16) might incorrectly predict a NS remnant signal instead of a prompt collapse. In practice, we do not have such EOS in our hadronic EOS sample but interesting examples are the EOS with hyperons [81] or with phase transitions to quark deconfined matter. We will discuss how to deal with these cases using a specific example below. Improvements in the modeling of the promptcollapse threshold and the waveform amplitudes for the short-lived cases are possible and will be considered in the near future as more and more accurate simulations become available.

\section{VALIDATION OF NRPM}

We compare the NRPM model to all nonspinning binaries in the CoRe database and to a "validation set" of ten simulations that were not employed for the fits of Sec. II B. The properties of the validation set are summarized in Table II. The simulations span the relevant ranges in $\boldsymbol{\theta}$, in particular covering the prompt-collapse and short-/longlived remnant cases. We compute the mismatch [82]

$$
\bar{F}=1-\max _{\phi_{0}, t_{0}} \frac{\left(h_{1}\left(\phi_{0}, t_{0}\right), h_{2}\right)}{\sqrt{\left(h_{1}, h_{1}\right)\left(h_{2}, h_{2}\right)}},
$$

based on the Wigner scalar product between two waveforms

$$
\left(h_{1}, h_{2}\right)=4 \Re \int_{f_{\min }}^{f_{\max }} \frac{\tilde{h}_{1}^{*}(f) \tilde{h}_{2}(f)}{S_{n}(f)} \mathrm{d} f,
$$

and assuming advanced LIGO design sensitivity [83-85] for the power-spectral-density (PSD) function $S_{n}(f)$ and $\left[f_{\min }, f_{\max }\right]=\left[f_{\mathrm{mrg}}, 4096 \mathrm{~Hz}\right]$. The value of $\bar{F}$ represents the loss in signal-to-noise ratio (squared) for waveforms that are aligned in time and phase. Additionally, we analyze time-domain phasing between the model and the NR waveforms.

Mismatches against the CoRe data used in the fits are shown in Fig. 4; the points relative to the validation set waveforms are shown as cyan triangle markers. The plot orders the binaries according to $\kappa_{2}^{\mathrm{T}}$. The largest mismatches are of order $\sim 0.65$ for $\kappa_{2}^{\mathrm{T}} \lesssim 200$, the smallest mismatches are of order $\sim 0.1$, and on average $\bar{F} \sim 0.3$. We recall that a mismatch $\bar{F}$ roughly corresponds to a fractional reduction in detection rate of $\sim 1-(1-\bar{F})^{3}$ for sources that are uniformly distributed in space $[88,89]$. Template banks for detection are usually constructed such that the maximum value of $\bar{F}$ across the bank is 0.03 , thus allowing for a 

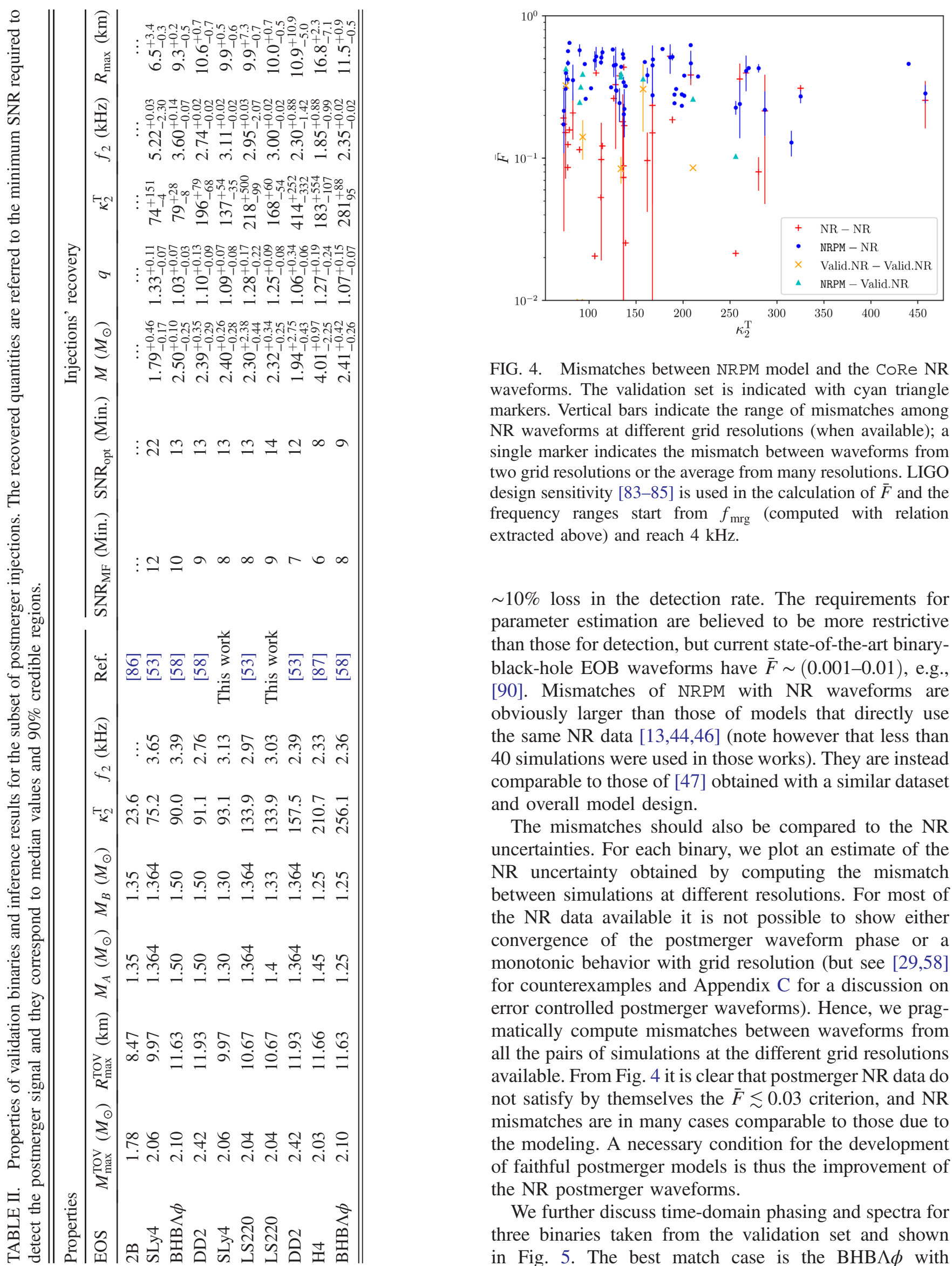

FIG. 4. Mismatches between NRPM model and the CoRe NR waveforms. The validation set is indicated with cyan triangle markers. Vertical bars indicate the range of mismatches among NR waveforms at different grid resolutions (when available); a single marker indicates the mismatch between waveforms from two grid resolutions or the average from many resolutions. LIGO design sensitivity [83-85] is used in the calculation of $\bar{F}$ and the frequency ranges start from $f_{\mathrm{mrg}}$ (computed with relation extracted above) and reach $4 \mathrm{kHz}$.

$\sim 10 \%$ loss in the detection rate. The requirements for parameter estimation are believed to be more restrictive than those for detection, but current state-of-the-art binaryblack-hole EOB waveforms have $\bar{F} \sim(0.001-0.01)$, e.g., [90]. Mismatches of NRPM with NR waveforms are obviously larger than those of models that directly use the same NR data $[13,44,46]$ (note however that less than 40 simulations were used in those works). They are instead comparable to those of [47] obtained with a similar dataset and overall model design.

The mismatches should also be compared to the NR uncertainties. For each binary, we plot an estimate of the NR uncertainty obtained by computing the mismatch between simulations at different resolutions. For most of the NR data available it is not possible to show either convergence of the postmerger waveform phase or a monotonic behavior with grid resolution (but see $[29,58]$ for counterexamples and Appendix $\mathrm{C}$ for a discussion on error controlled postmerger waveforms). Hence, we pragmatically compute mismatches between waveforms from all the pairs of simulations at the different grid resolutions available. From Fig. 4 it is clear that postmerger NR data do not satisfy by themselves the $\bar{F} \lesssim 0.03$ criterion, and NR mismatches are in many cases comparable to those due to the modeling. A necessary condition for the development of faithful postmerger models is thus the improvement of the NR postmerger waveforms.

We further discuss time-domain phasing and spectra for three binaries taken from the validation set and shown in Fig. 5. The best match case is the $\operatorname{BHB} \Lambda \phi$ with 

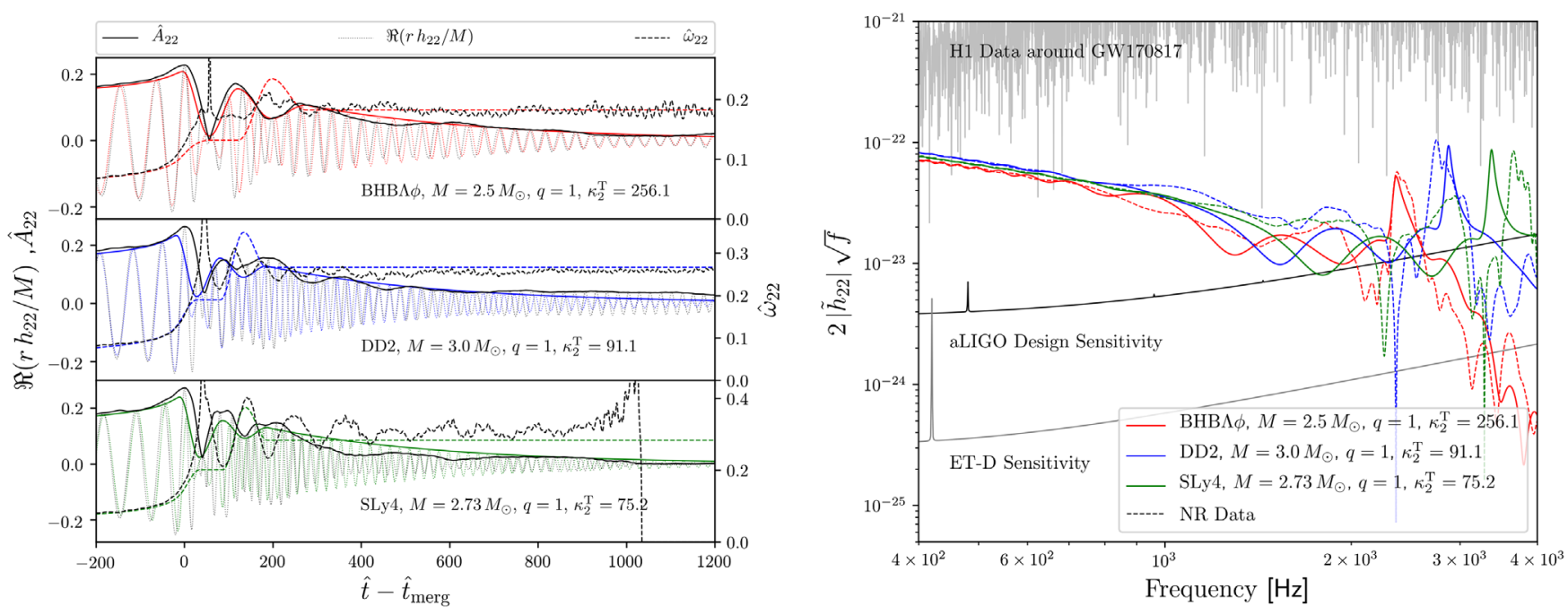

FIG. 5. Complete TEOBResums_NRPM $(2,2)$ waveforms and corresponding spectra. Left panel: Time-domain TEOBResumS $\operatorname{NRPM}(2,2)$ waveforms compared with selected NR hybrids around merger. From top to bottom, $\mathrm{BHB} \Lambda \phi M=(1.25+1.25) M_{\odot}$ is the best mismatch case, DD2 $M=(1.50+1.50) M_{\odot}$ represents an intermediate case and SLy4 $M=(1.364+1.364) M_{\odot}$ is the worst mismatch case. Right panel: Corresponding spectra from $400 \mathrm{~Hz}$ to $4 \mathrm{kHz}$ with sources located at $40 \mathrm{Mpc}$ and analytical power spectral densities of LIGO design [83-85] and Einstein Telescope [91,92].

$M=(1.25+1.25) M_{\odot}(\bar{F} \sim 0.1)$ for which the peak frequency $f_{2}=2358 \mathrm{~Hz}$ is well reproduced by the model (fit value $f_{2}^{\text {fit }}=2357 \mathrm{~Hz}$ ) and the waveform remains in phase for $\gtrsim 10 \mathrm{~ms}$ after merger. Phase differences at late times influence less the match since most of the energy is radiated earlier. The DD2 with $M=(1.50+1.50) M_{\odot}$ has a moderate match with NRPM. The model slightly overestimates $\hat{f}_{2}$ predicting $f_{2}^{\text {fit }}=2871 \mathrm{~Hz}$ instead of $f_{2}=2761 \mathrm{~Hz}$. Some significant dephasing is observed around $\hat{t} \sim 200$ for several cycles, and it is likely the main cause of the mismatch. The worst mismatch is obtained with the SLy4 with $M=(1.364+1.364) M_{\odot}$ that produces a short-lived remnant collapsing in $\sim 13 \mathrm{~ms}$. For this BNS the peak frequency is underestimated by the model $\left(f_{2}=3654 \mathrm{~Hz}\right.$ vs $f_{2}^{\text {fit }}=3367 \mathrm{~Hz}$ ). The NR frequency evolution has several oscillations and increases before collapse; these features are not modeled by NRPM. Consequently, the model has a poor match. Note that the $\hat{f}_{2 \pm 0}$ are rather well estimated in this case.

Inspection of other waveforms confirms that maintaining the phasing in the early postmerger signal is a key factor for the overall accuracy of the model. In addition, since the $\hat{f}_{2}$ fits of Sec. II are less accurate for small $\kappa_{2}^{\mathrm{T}}$, NRPM better describes the waveforms of BNS with larger $\kappa_{2}^{\mathrm{T}}$ corresponding to lower postmerger frequencies. Note that the latter are the most favored in low SNR detections. In other words, NRPM is more robust (uncertain) for a long-lived (short-lived) remnant, as expected. Finally, we test a simpler version of NRPM with the single frequency $\hat{f}_{2}$ and find that some short-lived data are actually better described by this simpler model which averages the frequency evolution.

\section{TIME-DOMAIN INSPIRAL-MERGER- POSTMERGER MODEL}

A model for the time-domain inspiral-mergerpostmerger (IMPM) waveform is obtained by smoothly attaching amplitude and phase of NRPM at the peak amplitude $\hat{A}_{\text {mrg }}$ of any time-domain inspiral-merger model. Currently, the only time-domain waveforms that can reproduce the merger peak amplitude are the effectiveone-body (EOB) ones. We thus use the tidal EOB model developed in $[86,90,93]$ and called TEOBResums.

The attachment is done at the amplitude peak as described in Sec. II A, but using the amplitude $\hat{A}_{\mathrm{mrg}}$, the merger frequency $\hat{\omega}_{\text {mrg }}$ and its derivative $\dot{\hat{\omega}}_{\text {mrg }}$ of the inspiral-merger waveform. Amplitudes $\hat{A}_{i}$ are then fixed by computing the ratios $\beta_{i}$. Examples of IMPM waveforms are shown in Fig. 5 and compared to NR waveforms. In order to perform a visual comparison, the NR and TEOBResumS_NRPM waveforms are aligned in phase and time at merger. The figure shows the smooth attachment at merger and the phase coherence of the postmerger completion. The figure also highlights that NRPM is more accurate for BNS with larger $\kappa_{2}^{\mathrm{T}}$, as discussed in Sec. III.

A quantitative measurement of the phase coherence is obtained by computing mismatches between the TEOBResumS_NRPM model and hybrid waveforms constructed joining TEOBResumS to NR data. We built such hybrid waveforms starting from a GW frequency of $50 \mathrm{~Hz}$ and for each BNS of the validation set. The mismatches are computed as functions of the lower cutoff frequency $f_{\min }$, which takes values from $50 \mathrm{~Hz}$ to $f_{\mathrm{mrg}}$, where the latter is obtained by the NR fits. Figure 6 shows the mismatches as a function of $f_{\min }$ for the validation set. Significant phase 


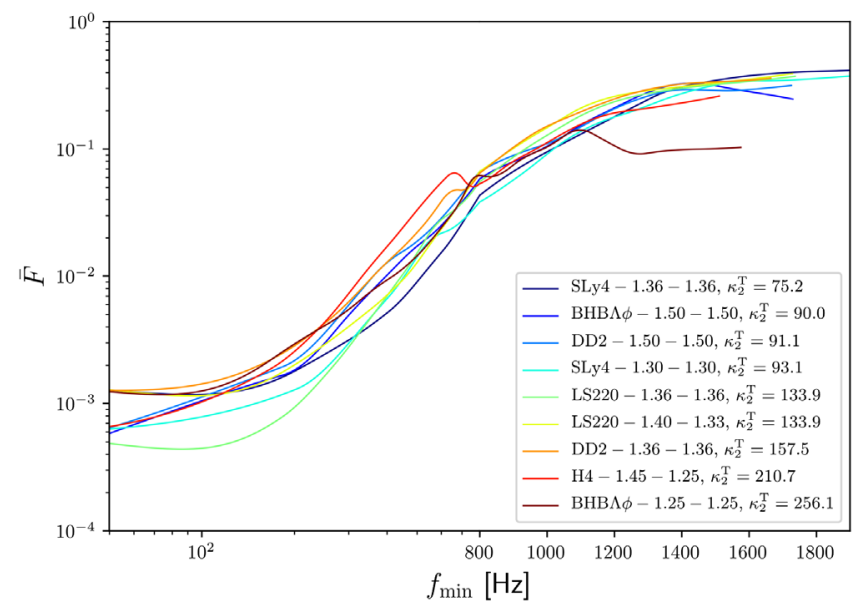

FIG. 6. Mismatches between hybrid waveforms (TEOBResumS+ NR) and the complete model TEOBResumS_NRPM as a function of lower cutoff frequency $f_{\min } \in\left[50 \mathrm{~Hz}, f_{\mathrm{mrg}}\right]$. The latter quantity is taken from the NR fits.

differences are accumulating between 500 and $800 \mathrm{~Hz}$ where the NR merger is attached. The last point of each line corresponds to the mismatch between NRPM and NR; typical values are $\bar{F} \lesssim 0.3$ with a minimum $\bar{F} \sim 0.1$ consistently with what was discussed in Sec. III.

\section{INJECTION STUDIES}

To demonstrate the applicability of NRPM in the context of Bayesian GW data analysis we consider a set of experiments in which known signals are injected using zero-noise configuration and recovered using standard Bayesian inference techniques. The experiments aim at addressing the following questions:

(A) At which SNR can NRPM detect a PM signal?

(B) Is it possible to infer whether the merger remnant collapsed to a $\mathrm{BH}$ or was a NS using the IMPM model?

(C) What constraints can be set on the NS minimal radius from the PM analysis solely?

(D) Is it possible to infer the EOS stiffness at the extreme densities reached in the NS remnant using the IMPM signal?

Given data $d$ and hypothesis $H$, the posterior distribution of the parameters $\Theta$ is defined from Bayes's theorem,

$$
p(\boldsymbol{\Theta} \mid d, H)=\frac{p(d \mid \boldsymbol{\Theta}, H) p(\boldsymbol{\Theta} \mid H)}{p(d \mid H)},
$$

where $p(\boldsymbol{\Theta} \mid H)$ is the prior distribution for the parameters $\boldsymbol{\Theta}$ and $p(d \mid \boldsymbol{\Theta}, H)$ is the likelihood function. For a single detector $i$, the likelihood is defined as

$$
\log p_{i}(d \mid \boldsymbol{\Theta}, H) \propto-\frac{1}{2}\left(d-h_{\boldsymbol{\Theta}}, d-h_{\boldsymbol{\Theta}}\right)_{i}
$$

where $h_{\boldsymbol{\Theta}}$ is the GW template, which depends on the parameters $\boldsymbol{\Theta}$. For a detector network it is obtained by multiplying the likelihood of the single detectors. The term $p(d \mid H)$ is the evidence and it can be computed as the marginalization of the likelihood function over the entire parameter space.

We perform two sets of experiments using the amplitude sensitivity densities (ASD) of the three Advanced LIGO [83-85] and Advanced Virgo detectors [94]. In the first set, we inject nine postmerger signals of the validation set reported in Table II placing the source at 2, 3, 4, 5, 6, 7, $8 \mathrm{Mpc}$ and located at right ascension and declination $(\alpha, \delta)=(0,0)$ with angle of view $\imath=0$, polarization angle $\psi=0$ and sampled at $8192 \mathrm{~Hz}$. In the injections, we apply a Tukey window at merger in order to isolate the postmerger signal and remove the contributions from the inspiral. The distances approximately correspond to postmerger SNRs from 4 to 16, with the exact values depending on the particular BNS. The injected NR signals are recovered with NRPM by analyzing the frequencies $[1024,4096] \mathrm{Hz}$ and fixing the sky location of the source. Inference is performed on the extended set of parameters

$$
\boldsymbol{\Theta}=\left(M_{A}, M_{B}, \Lambda_{A}, \Lambda_{B}, D_{L}, \psi, t_{0}, \phi_{0}\right),
$$

where $\left(t_{0}, \phi_{0}\right)$ are the time shift and the merger phase, respectively, and $\psi$ is the polarization angle. In this paper we prescribe the collapse threshold as $\kappa_{\mathrm{thr}}^{\mathrm{T}}=70$; for more general analysis the parameter can be included into $\boldsymbol{\Theta}$. We also use the $\alpha$ parameter in Eq. (11) as estimated from the NR fits but, as discussed in Sec. II B 1, uncertainties on the $\alpha$ fit can lead to incorrect distance estimates. In future analysis the effect of promoting $\alpha$ to an inference parameter should be explored, effectively allowing for a more agnostic analysis.

The posterior distributions of other parameters are recovered using their definitions or from the fits in the case of peak frequencies. Priors are set on chirp mass, mass ratio and $\Lambda_{A, B}$, that are bounded to $\mathcal{M}_{c} \in[0.5,2.2] M_{\odot}$, $q \in[1,1.5]$ and $\Lambda_{A, B} \in[50,5000]$. The prior distributions are uniform in the individual components $M_{A, B}$ and $\Lambda_{A, B}$. Bayesian inference is performed with the nested sampling algorithm [95] as implemented in the LALInference software package [96-98].

In the second set, we inject hybrid waveforms and we recover with either the IM model or the IMPM model. Specifically, we use the nonspinning surrogate of TEOBResum developed in [99] and refer to the IM (IMPM) model as TEOBResum_ROM (TEOBResum_ROM_NRPM). The choice of the priors is identical to the previous cases, except for the chirp mass for which we use a smaller range $\mathcal{M}_{c} \in[1,2.2] M_{\odot}$, and the frequency range analyzed is $[50$, 4096] Hz. We note that the injection labeled as 2B $M+$ $(1.35+1.35) M_{\odot}$ is a prompt-collapse signal. NRPM does not include a template for these types of sources and then 

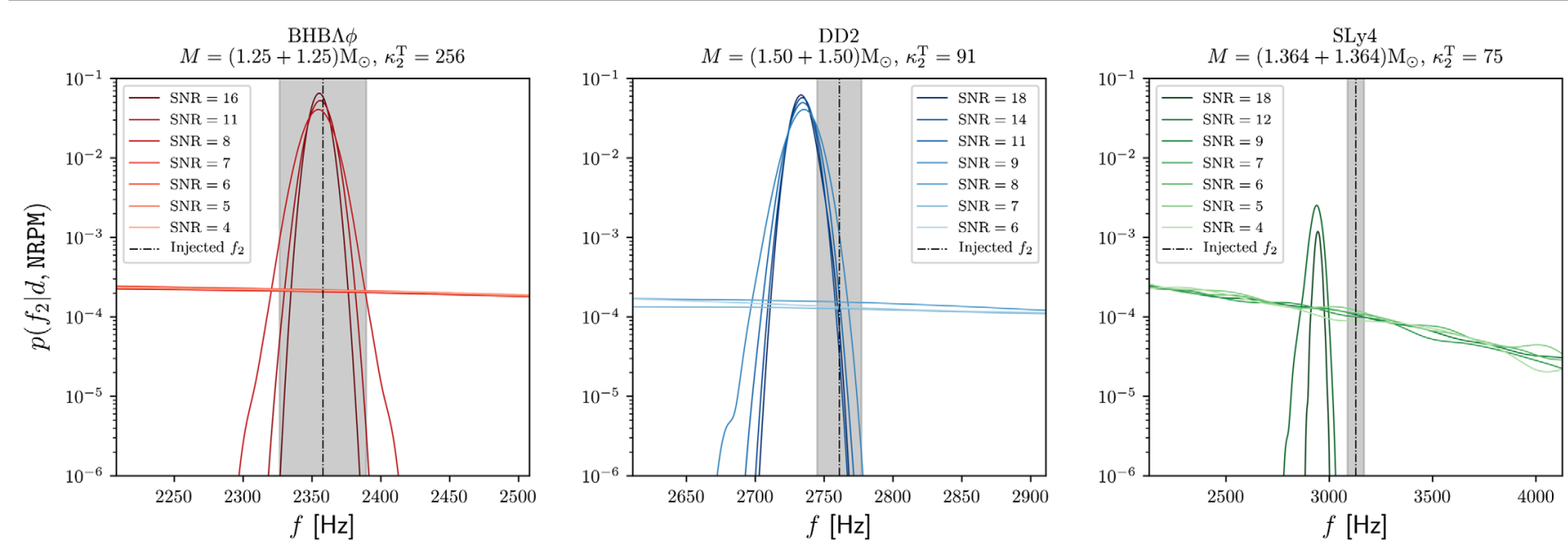

FIG. 7. Marginalized posterior distributions of $f_{2}$ for three injected cases at different SNRs: the first case, $B H B \Lambda \phi$ $M=(1.25+1.25) M_{\odot}$, is a case where the peak frequency is well recovered and this is also supported by the low mismatch between the NRPM model and the injected signal. In the second case, DD2 $M=(1.50+1.50) M_{\odot}$, we can see that for high SNRs biases appear systematically and the recovered peak is below the injected one. The third case, SLy $4 M=(1.364+1.364) M_{\odot}$, shows a bimodal distribution: a dominant peak appears at frequency $\sim 5.2 \mathrm{kHz}$ (beyond the Nyquist limit, not in the plot) while the secondary peak is close to the injected value. The primary peak is compatible with the frequency $f_{2-0}$ aliased at high frequencies.

this waveform is excluded from the detectability application, but it is included in the second set of injections (Sec. V B).

Considering a GW170817-like source, an optimal SNR $\sim 3$ could be achieved by the Advanced LIGOVirgo detectors at design sensitivity, while SNR $\sim 10$ is expected to be achieved by third-generation detectors. From now on, the SNR value we quote is the maximum value coming from the matched-filtered SNR computation between the NRPM model and the injected signal.

\section{A. Postmerger detectability}

We discuss the results of the first set of injections employing only PM signals and NRPM. The matched filtering analysis of the validation set gives evidence of postmerger signals starting from network SNR 8-9. The latter correspond to source distances of 4-6 Mpc. We find that statistical errors are larger than systematic uncertainties at $\mathrm{SNR} \lesssim 12$ but the two become comparable for higher SNRs.

The parameters recovered by the analysis at the minimal SNR are reported in Table II. For most of the cases, the posterior distributions of the physical parameters include the injected values within the $95 \%$ confidence regions. However, some cases show degeneracies among the model's parameters. In general, the largest discrepancies in the recovered parameters are induced by the inaccuracy of the NR frequency fit for the particular BNS. The posterior distributions for $f_{2}$ for three exemplary cases at different SNRs are shown in Fig. 7. NRPM recovers the correct peak frequency within the uncertainties for all the injected binaries except for the DD2 $M=(1.50+1.50) M_{\odot}$ which will be discussed in Sec. V D.
For the injection $\mathrm{BHB} \Lambda \phi M=(1.25+1.25) M_{\odot}$, the estimation of the parameters with NRPM is in agreement with the injected properties. The posterior distributions are unimodal and centered around the injected value. In this case, the model is able to reconstruct the spectrum of the signal and this fact is also motivated by the low mismatch between this waveform and the model.

A difficult case is SLy4 $M=(1.364+1.364) M_{\odot}$ for which the values of the masses and $\kappa_{2}^{\mathrm{T}}$ are underestimated to compensate the smaller values of $\hat{f}_{2}$ estimated from the NR fits, and to obtain a signal matching the injection $\left(f_{2} \propto M^{-1}\right)$. Moreover, the marginalized posterior distribution of $f_{2}$ has a bimodality. For this signal, $f_{2}$ is at the edge of the frequency range where the sensitivity is smaller and the recovery with NRPM promotes the subdominant peak $f_{2-0}$ as the main frequency, especially for high SNR. However, the $f_{2-0}$ is aliased to high frequencies and the maximum of the marginalized posterior distribution of $f_{2}$ is well above the Nyquist frequency of $\sim 4 \mathrm{kHz}$ (not shown in the plot). The secondary maximum of the distribution is compatible with the injected value within the uncertainties.

Another interesting case is $\mathrm{BHB} \Lambda \phi \quad M=(1.50+$ $1.50) M_{\odot}$ : this postmerger signal is very short and the remnant collapses after $\sim 3 \mathrm{~ms}$. As a consequence, the frequency evolution is not trivial and none of the spectrum peaks is relevantly dominant, since the remnant evolves towards collapse. Then, the recovered $f_{2}$ peak is overestimated while the $f_{2-0}$ peak is correctly captured $\left(f_{2-0}^{\mathrm{inj}}=\right.$ $2.48 \mathrm{kHz}$ vs $f_{2-0}^{\text {rec }}=2535_{-48}^{+40} \mathrm{~Hz}$ at SNR 11).

In general, we observe for some cases a shift in the recovered value of the total mass $M$ : this parameter strongly correlates with the position of the frequency peak and with its amplitude in the frequency domain. The latter quantities 
are also determined by the damping time in Eq. (11), whose behavior is not well captured by NR fits (Table I). These uncertainties propagate during the parameter estimation routine and the results are biased. However, these effects could be avoided including $\alpha$ into $\boldsymbol{\Theta}$. Moreover, this estimation can be inferred with high accuracy from the inspiral measurement at these SNRs.

\section{B. Inferring prompt collapse}

We discuss the results of the second injection set focusing on two different BNS: 2B $M=(1.35+$ $1.35) M_{\odot}$ which ends in a prompt collapse, and $\mathrm{BHB} \Lambda \phi$ $M=(1.25+1.25) M_{\odot}$ for which the outcome is a longlived remnant (see Fig. 5). In the context of Bayesian analysis, a natural approach for prompt-collapse inference is to perform model selection between inspiral-merger and inspiral-merger-postmerger models for the given data. In the case of prompt collapse, the IM model should be favored with respect to the IMPM one, while in the case of a long-lived NS remnant should be the opposite. Note that this analysis relies on the existence of a coherent model for the full spectrum (modeling the IMPM phases), as the one proposed here.

Specifically, we perform model selection using the Bayes's factor $\mathcal{B}$, which quantifies the agreement of two different competitive hypotheses, $H_{A}$ and $H_{B}$, with the data. The Bayes's factor is defined as the ratio of the two posterior probabilities; however it is possible to prove that it can be computed as the ratio of the evidences,

$$
\mathcal{B}_{B}^{A}=\frac{p\left(d \mid H_{A}\right)}{p\left(d \mid H_{B}\right)} .
$$

If $\mathcal{B}_{B}^{A}>1(<1)$, the hypothesis $A(B)$ is favored. In our case, the competitive models are TEOBResum ROM for the IM, and TEOBResum_ROM_NRPM for the IMPM. For this test we remove the constraint given by $\kappa_{\text {thr }}^{\mathrm{T}}$ on NRPM.

We inject the $2 \mathrm{~B}$ and $\mathrm{BHB} \Lambda \phi$ signals using a SNR $\sim 12$, sufficient to detect the postmerger signal with NRPM. We recover with and without attaching NRPM model at merger. The values of the Bayes's factors obtained are reported in Table III. The algorithm is able to distinguish whether the remnant has undergone prompt collapse or not: the Bayes's factor for $2 \mathrm{~B} M=(1.35+1.35) M_{\odot}$ correctly

TABLE III. Evidences computed for the prompt-collapse inference. The uncertainties are estimated with the criterion introduced in Ref. [95]. The label "noise" refers to the template identically equal to zero.

\begin{tabular}{lccc}
\hline \hline Injection & $\log \mathcal{B}_{\text {noise }}^{\text {IM }}$ & $\log \mathcal{B}_{\text {noise }}^{\text {IMPM }}$ & $\log \mathcal{B}_{\text {IM }}^{\text {IMP }}$ \\
\hline 2B $M=(1.35+1.35) M_{\odot}$ & $124845_{-1}^{+1}$ & $124775_{-1}^{+1}$ & $-70_{-2}^{+2}$ \\
BHB $\Lambda \phi M=(1.25+1.25) M_{\odot}$ & $107116_{-1}^{+1}$ & $107306_{-1}^{+1}$ & $190_{-2}^{+2}$ \\
\hline \hline
\end{tabular}

favors the model without postmerger $\left(\log \mathcal{B}_{\mathrm{IM}}^{\mathrm{IMPM}}=\right.$ $\left.-70_{-2}^{+2}\right)$. Similarly, for $\mathrm{BHB} \Lambda \phi M=(1.25+1.25) M_{\odot}$ the presence of a postmerger signal is favored with respect to the prompt-collapse case $\left(\log \mathcal{B}_{\mathrm{IM}}^{\mathrm{IMPM}}=190_{-2}^{+2}\right)$.

We point out that numerical relativity simulations indicate that in prompt-collapse waveforms a signal, not described by EOB waveforms, is present after the amplitude peak. We find that the SNR contribution of this short, $\lesssim 2 \mathrm{~ms}$, postmerger signal in the full spectrum of $2 \mathrm{~B} M=$ $(1.35+1.35) M_{\odot}$ is below $4 \%$.

\section{Constraints on NS minimal radius}

As shown in Table II, at the minimal SNR the inference on $f_{2}$ delivers a result accurate at $2-16 \%$ (two-sigma). Using the EOS-independent relation of $f_{2}\left(R_{1.6}\right)$ from [45], this measurement could be translated into an estimate of the radius of a nonrotating equilibrium star of mass $1.6 M_{\odot}$ $\left(R_{1.6}\right)$ with an uncertainty of $\sim 1.5 \mathrm{~km}$. In a real scenario this is not particularly interesting since the radius (or equivalently the tidal parameters, $R \sim \tilde{\Lambda}^{1 / 5}[100,101]$ ) will be known with an accuracy at least 100 times better from the inspiral-merger analysis. We find from our runs that inspiral-merger inference at the minimal postmerger SNR delivers $\delta \tilde{\Lambda} / \Lambda \sim 0.04$ and $\delta R / R \sim 0.008$.

More interesting is to explore constraints on the radius of the maximum mass (most compact) nonrotating equilibrium NS $R_{\max }^{\mathrm{TOV}}$ [48], since the latter corresponds to the largest matter densities that can be reached for a given EOS. Using the CoRe NR data, we find an approximate relation in the form

$$
\begin{aligned}
\hat{R}_{\max }\left(\hat{f}_{2}\right)= & (5.81 \pm 0.13)-(123.4 \pm 7.2) \hat{f}_{2} \\
& +(1121 \pm 99) \hat{f}_{2}^{2}
\end{aligned}
$$

where $\quad \hat{R}_{\max }=R_{\max }^{\mathrm{TOV}} / M$ and fitting $\chi^{2}=7.4 \times 10^{-5}$. Measurements of PM signals at the minimum SNR deliver an estimation of $R_{\max }$ accurate at the $\sim 8 \%$ level. The fit uncertainty is smaller than statistical error at SNR 8, and they become comparable for SNR 11. Figure 8 shows the data and fit for Eq. (23) together with examples of the posteriors for $R_{\max }^{\mathrm{TOV}}$. The latter can be inferred with an uncertainty of $\sim 1 \mathrm{~km}$.

Some cases show biased results: for DD2 $M=(1.50+$ $1.50) M_{\odot}$ the expected maximum radius underestimates the $R_{\max }^{\mathrm{TOV}}$ predicted by the related EOS, while for $\mathrm{H} 4 M=$ $(1.45+1.25) M_{\odot}$ the recovery overestimates the relative value. These shifts are coherent with the erroneous estimation of the total mass $M$, previously discussed in Sec. VA.

\section{Inferring EOS stiffness at extreme densities}

We demonstrate the possibility of investigating the EOS stiffness at extreme densities using the postmerger GW observations and NRPM. We discuss the specific case of 




FIG. 8. Characteristic postmerger frequency $\hat{f}_{2}$ against $\hat{R}_{\max }$ extracted from NR data for different EOS. The black solid line represents the fit with its $90 \%$ credible region. The right panel shows the marginal posterior distributions of $\hat{f}_{2}$ for three selected injections while the top panel shows the respective $\hat{R}_{\max }$ marginal distributions.

EOS $\mathrm{BHB} \Lambda \phi$ and DD2, previously simulated by some of the authors [58]. The $\mathrm{BHB} \Lambda \phi$ EOS is identical to DD2 except that at densities $\rho \gtrsim 2.5 \rho_{0}$ (where $\rho_{0}$ is the nuclear density) it softens due to the formation of $\Lambda$-hyperons. Inspiral-merger $\mathrm{GW}$ signals from binaries described by the two EOS and $M \lesssim 3 M_{\odot}$ are indistinguishable since the individual NSs have maximal densities $\rho \lesssim 2.5 \rho_{0}$, similar compactnesses and tidal parameters (same $\kappa_{2}^{\mathrm{T}}$, Fig. 9).

We consider two pairs of binaries: a "low mass" with the $M=2.5 M_{\odot}$ pair and "high mass" with the $M=3 M_{\odot}$ pair. The individual NS of the low-mass BNS have central density $\rho \approx 2.35 \rho_{0}$ and there are essentially no $\Lambda$-hyperons at these densities in the BHB $\Lambda \phi$ EOS. The BNS remnants relative to the latter EOS reach approximately $\rho \approx 2.80 \rho_{0}$ at which $\mathrm{BHB} \Lambda \phi$ differs from the DD2 EOS. The GW postmerger signals have very similar $f_{2}$ frequencies, but they are in principle distinguishable at sufficiently high SNR [58]. The individual NS of the high-mass BNS have $\rho \approx 2.75 \rho_{0}$; the presence of $\Lambda$-hyperons significantly affects the postmerger dynamics. The DD2 binary produces a remnant surviving for $\gtrsim 20 \mathrm{~ms}$ while the $\mathrm{BHB} \Lambda \phi$ binary collapses within $\sim 2 \mathrm{~ms}$ as a result of the EOS softening. The postmerger signals are consequently very different, as illustrated in Fig. 9 (bottom panel).

Figure 10 shows $68 \%$ and $95 \%$ confidence regions of the marginal posterior distributions in the $\left(f_{2}, \kappa_{2}^{\mathrm{T}}\right)$ plane as a summary plot of the inference results at two different SNR; the left panels refer to the low-mass BNSs, right panels to high masses. The postmerger analysis of the low-mass
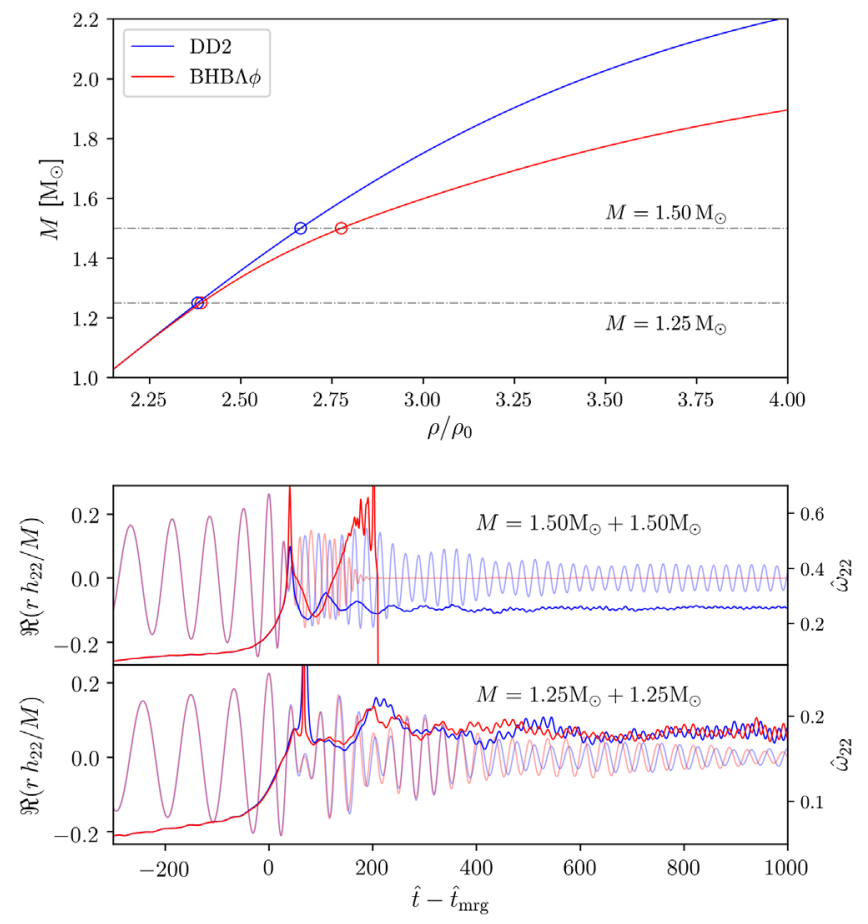

FIG. 9. Binary neutron stars described by the $\mathrm{BHB} \Lambda \phi$ and the DD2 EOS and simulated signals [58]. Top: Mass of individual spherical equilibrium NS as a function of the central density. Markers refer to simulated BNS. Bottom: Real part of the $(2,2)$ waveforms for BNSs with mass $M=(1.50+1.50) M_{\odot}$ and $M=(1.25+1.25) M_{\odot}$.

BNSs returns the injected values and it agrees with the inference from the inspiral analysis. At SNR 16 some deviations are visible in the posteriors' distribution indicating that such small differences might be detectable with more accurate models and measurements.

The postmerger analysis of the high-mass DD2 $M=$ $(1.50+1.50) M_{\odot}$ shows that the injected frequency is correctly captured by the recovery, while the frequency estimated from the inspiral-merger analysis and the fit is slightly overestimated (as expected, cf. Fig. 5). As a consequence of this, the $\kappa_{2}^{\mathrm{T}}$ posterior from the postmerger analysis is not compatible with the inspiral measurement at the minimal SNR (upper right panel). However, at higher SNR the correct $\kappa_{2}^{\mathrm{T}}$ is consistently recovered within the $68 \%$ confidence region (lower right panel).

For the $\mathrm{BHB} \Lambda \phi$ high-mass $M=(1.50+1.50) M_{\odot}$ case, we find instead inconsistencies between $\kappa_{2}^{\mathrm{T}}$ and $f_{2}$ posteriors computed from the IM and PM analysis respectively. The postmerger analysis returns a $f_{2}$ higher than the injected signal, especially at high SNR. At the same time, the $\kappa_{2}^{\mathrm{T}}$ distribution from the postmerger analysis is shifted towards lower values at larger SNR and rails against the prompt-collapse value $\kappa_{2}^{\mathrm{T}} \sim 70$, significantly departing from the inspiral measurement $\kappa_{2 \mathrm{IM}}^{\mathrm{T}}=93_{-3}^{+2}$. The templated-analysis of the postmerger clearly tries to fit the higher frequencies of the signal $\left(f_{2}=3.39 \mathrm{kHz}\right)$ and the 

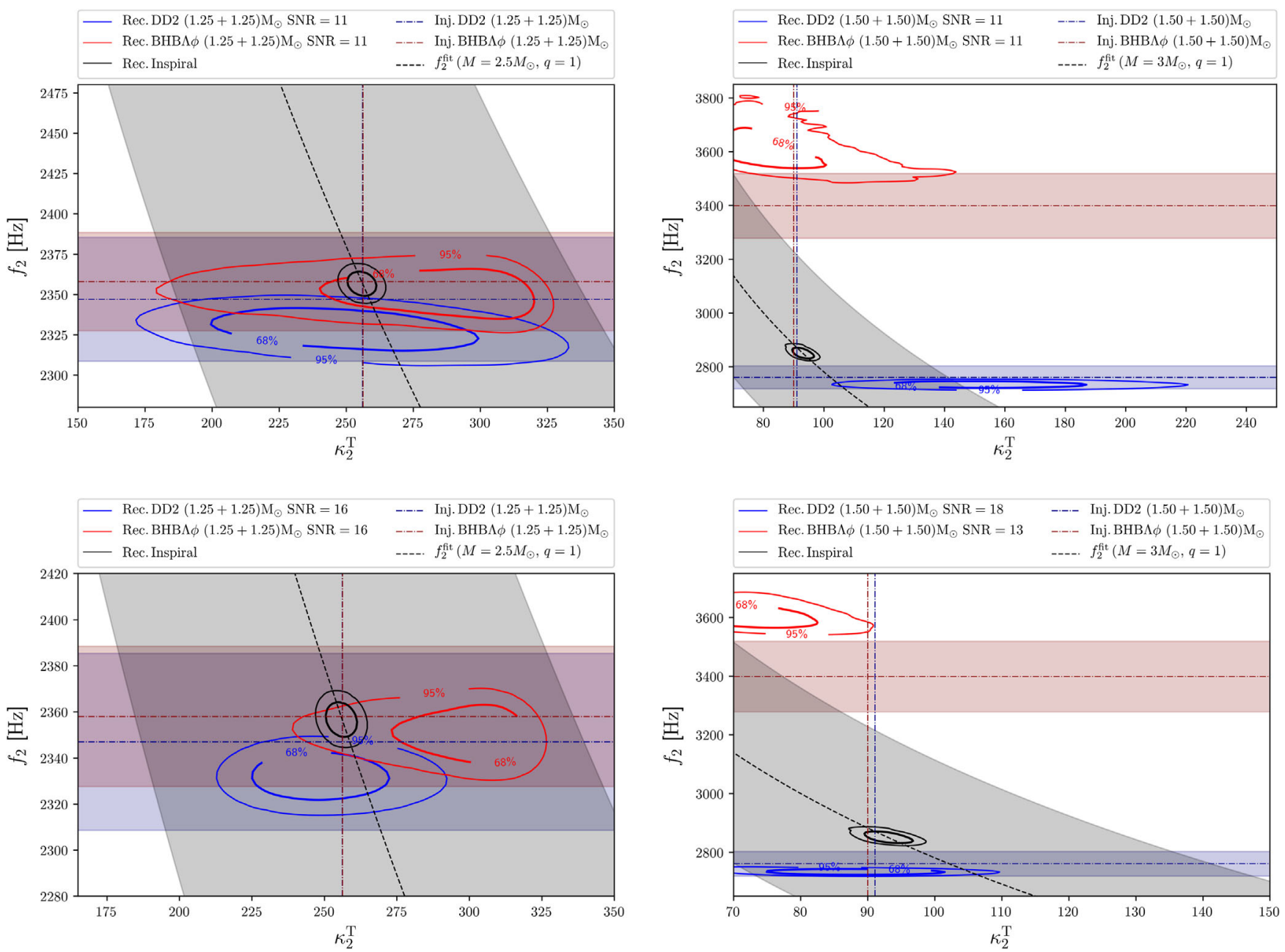

FIG. 10. Inference of EOS properties at extreme densities. Left panel: Marginalized posterior distributions of $f_{2}$ and $\kappa_{2}^{T}$ for the lowmass cases (SNR 11 and 16). The postmerger posteriors agree with the value predicted by the fit and with the measurement from the inspiral. Right panel: Marginalized posterior distributions of $f_{2}$ and $\kappa_{2}^{\mathrm{T}}$ for the high-mass cases (SNR 11 and higher). The panels also shows $f_{2}\left(\kappa_{2}^{\mathrm{T}}\right)$ fits related to the injected values with the associated $90 \%$ credible regions. The uncertainties associated to the injected $f_{2}$ are the widths of the relative peaks in the frequency domain.

short postmerger signal collapsing to $\mathrm{BH}$. The high frequencies of the $\mathrm{BHB} \Lambda \phi$ binary are incompatible with the quasiuniversal of the NRPM model, due to the physical softening of the EOS. Thus, the analysis of the postmerger signal effectively implies a softer EOS than the analysis of the inspiral implies.

In a real GW measurement the difference in the inferences of $\kappa_{2}^{\mathrm{T}}$ (PM vs IMPM results in the high-mass BHB $\Lambda \phi$ case) will give an indication of the EOS softening at densities larger than those of the individual NS. The constraint follows from the breaking of the quasiuniversal relation $f_{2}\left(\kappa_{2}^{\mathrm{T}}\right)$, but the latter does not necessarily imply the presence of new degrees of freedom or phase transitions (cf. [59]). The case studies suggest that a measurement at $\mathrm{SNR} \gtrsim 11$ leads to deviations from the expected values larger than the $90 \%$ credible regions, which is sufficient to make a prediction with significance greater than the onesigma level.

\section{CONCLUSION}

NRPM is a time-domain analytical model for postmerger waveforms with minimal, but physically motivated, parameters describing the morphology of the postmerger waveforms in the binary (intrinsic) parameter space defined by Eq. (6). Combined with inspiral-merger effective-one-body waveforms, it forms an approximant coherent in phase on the full frequency range observed by ground-based interferometers. Future directions in the modeling of the postmerger waveform will include the extension of the CoRe database and the application of statistical/data reduction methods for the construction of more accurate and reliable templates [13,44]. Central goals for numerical simulations are a better characterization of the prompt-collapse threshold and errorcontrolled postmerger waveforms with microphysical EOS and unequal masses. 
The current accuracy of the model seems sufficient for the recovery of signals with postmerger SNR $\sim 8.5$. These results, although for a limited set of injections, suggest that Bayesian template-based analyses of the postmerger require higher SNRs than the morphology-independent analysis $[14,42]$. The latter references claim that about $90 \%$ of the signal can be reconstructed at $\mathrm{SNR} \sim 5$. Although a direct comparison of a detectability threshold in the two types of methods is difficult, the apparent higher requirement in SNR of the template-based methods is unsurprising, since these methods attempt to model and recover the entire postmerger signal, as opposed to only capturing its dominant feature. Additionally, the uncertainties associated with numerical relativity simulations and with the related fits significantly contribute to the mismatch (averaging to $\bar{F} \sim 0.3$, Fig. 4) and therefore affect the detectability in the template-based method. An advantage of our method is the possibility of performing coherent analysis of the inspiral-merger-postmerger spectrum. We showed that a straightforward application of our models in the context of Bayesian model selection is the inference of promptcollapse/remnant star scenarios.

The quasiuniversal (approximately EOS-independent) relations established in this paper extend previous results and can be employed also with other modeling techniques. On the one hand, they are the key to building waveform models because they connect the main signal's features with the binary (progenitor NS) properties. On the other hand, their direct use in constraining the EOS is not always relevant. GW measurements of $R_{1.6}$ or $\kappa_{2}^{\mathrm{T}}$ from $f_{2}$ will not add significantly new information about the EOS at extreme densities because the inspiral signals of the same sources will deliver more accurate measurements (stronger EOS constraints) of the same quantities. For example, the NS radius at fiducial masses would be known at $\lesssim 10$ meters precision from inspiral measurements against the kilometer precision of postmerger measurement, with the meter precision being more accurate than any quasiuniversal relation known to date.

With this in mind, we have explored a recalibration [Eq. (23)] of the relation $R_{\max }^{\mathrm{TOV}}\left(f_{2}\right)$ connecting the peak frequency to the radius of the most compact NS [48]. The latter effectively corresponds to the maximal NS central densities, and it is unlikely that such NS will be components of a binary system. A single postmerger signal at minimal SNR would deliver $R_{\max }^{\mathrm{TOV}}$ within an error of $\sim 8 \%$ (few kilometers). Assuming no systematic effect from the template-based inference, the uncertainty on $R_{\max }^{\mathrm{TOV}}$ at minimal SNRs is comparable.

A second constraint of the EOS at extreme densities could come from the identification of softness effects. We demonstrated that inconsistencies in the tidal polarizability and in the characteristic frequency peak inferred independently from the inspiral-merger and postmerger analysis can indicate EOS stiffening/softening at densities $\sim 3-5 \rho_{0}$ already at minimal SNR for detection. Note that this approach has similarities to the inspiral-merger-ringdown consistency tests performed on BH signals [102-105]. It is important to stress that no specific physical mechanism determining the softening/stiffening is modeled in NRPM (nor in the NR relations), but the information follows from the breaking of the specific quasiuniversal relation. An interesting development would be to perform model selection on different postmerger models, should NR quasiuniversal models based on specific EOS parametrization/ families become available.

\section{ACKNOWLEDGMENTS}

The authors thank the LIGO-Virgo matter and postmerger group for discussions. M. B., S. B., and F.Z. acknowledge support by the EU H2020 under ERC Starting Grant No. BinGraSp-714626. D. R. acknowledges support from a Frank and Peggy Taplin Membership at the Institute for Advanced Study and the Max-Planck/ Princeton Center (MPPC) for Plasma Physics (NSF Grant No. PHY-1804048). Parameter estimation was performed on the Virgo "Tullio" server at Torino supported by INFN and on LIGO Laboratory supercomputers, supported by NSF Grants No. PHY-0757058 and No. PHY-0823459. Numerical relativity simulations were performed on the supercomputer SuperMUC at the LRZ Munich (Gauss project pn56zo); on the supercomputer Marconi at CINECA (ISCRA-B project number HP10B2PL6K and HP10BMHFQQ); on the supercomputers Bridges, Comet, and Stampede (NSF XSEDE allocation TG-PHY160025); on NSF/NCSA Blue Waters (NSF AWD-1811236); on the ARA cluster at Jena FSU.

\section{APPENDIX A: QUASIUNIVERSAL RELATIONS}

We collect in this Appendix various plots of quasiuniversal relations for amplitudes and times. Figure 11 shows amplitude and time fits extracted from NR data of the CoRe Collaboration and implemented in the NRPM model. The robustness of those relations is further demonstrated using the independent data from SACRA code [51] that were not used in this work. To this purpose Fig. 12 shows a comparison between the $f_{2}$ extracted from the SACRA catalog [51] and the CoRe data and fits.

We give a heuristic justification of the quasiuniversal relations (employed here and elsewhere to summarize NR information) and of the choice of the parametrization. The discussion follows from the original argument given in [86].

While the choice of the parameter in Eq. (12) should be primarily considered as an operative choice, it can be in part justified based on perturbative arguments. In the effective-one-body (EOB) description of the two-body dynamics or, equivalently in this case, in the postNewtonian formalism, the interbinary potential $A(u)$, 

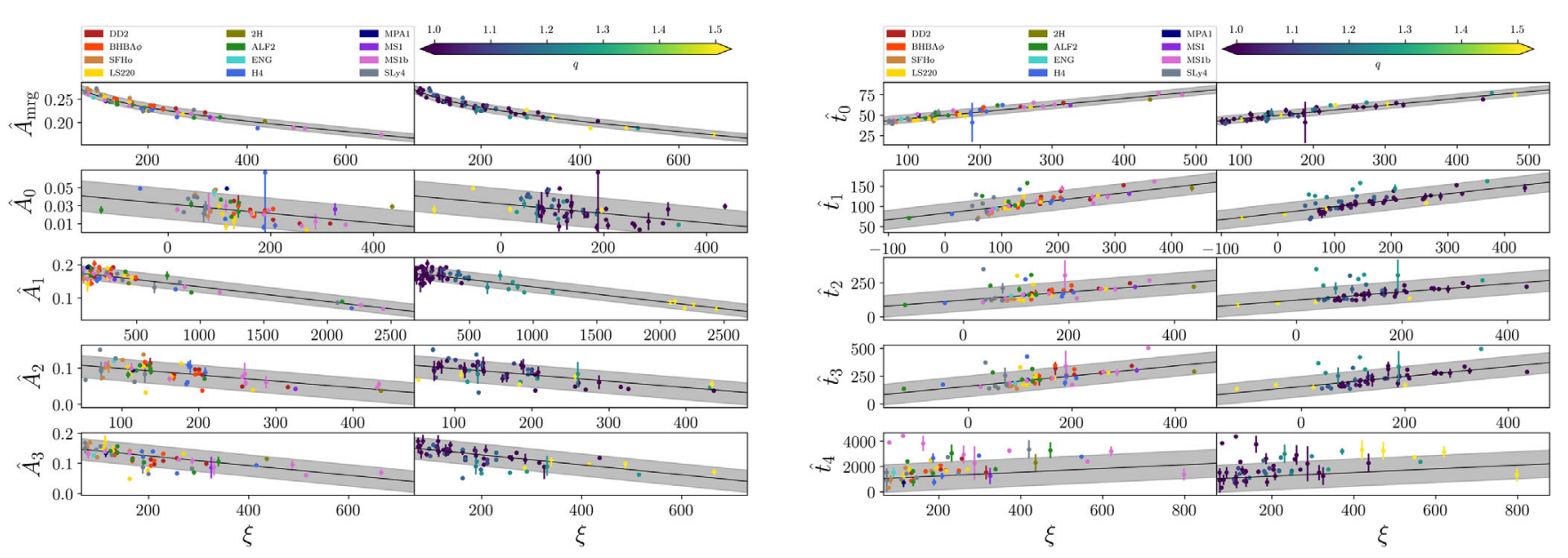

FIG. 11. Characteristic amplitude and time information from NR simulations. Markers represent the quantities extracted from the NR data; the black lines are the fits with their $90 \%$ credible regions. All upper panels show the same data; the colors on the left panel correspond to the EOS variation, and on the right panel to the mass ratio. Note that we impose a lower bound for $\hat{A}_{0}$ equal to zero for all those values of $\xi$ that lead to negative results in the fits.

where $u=G M /\left(r c^{2}\right)$, is the main quantity which describes the binary dynamics. The radial force governing the circular motion is given by

$$
\frac{d A}{d r}=-u^{2}\left(-2+\hat{a}_{0}^{\prime}(\nu, u)+\hat{a}_{T}^{\prime}\left(\kappa_{\ell}^{A}, \nu, u\right)\right),
$$

where $\hat{a}_{0}$ and $\hat{a}_{T}$ are the point-mass and the tidal corrections to the Newtonian term respectively (we neglect here spin interactions). The tidal contribution is in general parametrized by the multipolar tidal polarizability coefficients $\kappa_{\ell}^{A}$ of each NS [64]. At leading order in $1 / c^{2}$ the two terms above read

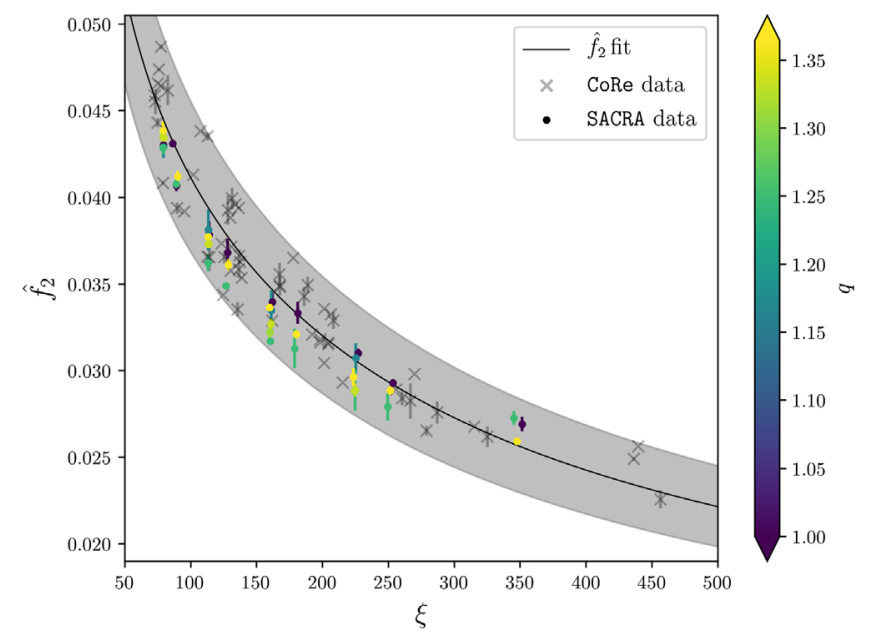

FIG. 12. Postmerger frequencies $f_{2}$ from the CoRe database (gray crosses) and the SACRA catalog [106,107] (colored dots), averaged on different resolutions. The black solid line is the quasiuniversal relation for $\hat{f}_{2}$ extracted from CoRe data with its $90 \%$ credible region.

$$
\hat{a}_{0}(\nu, u) \propto \nu u^{2}, \quad \hat{a}_{T}\left(\kappa_{\ell}^{A}, \nu, u\right) \propto-\kappa_{2}^{\mathrm{T}} u^{3} .
$$

Hence, finite mass-ratio and tidal effects are parametrized at leading order by $\nu$ and $\kappa_{2}^{\mathrm{T}}=\kappa_{2}^{A}+\kappa_{2}^{B}$. Note that the two contributions are associated with different powers in $u$ (different post-Newtonian orders) and have opposite sign.

As noted in [86], in the strong-field regime (where the expansion above is not accurate), and in particular close to the EOB last stable orbit $u \sim 0.14$, the tidal term $\hat{a}_{T}$ can become numerically comparable to $\hat{a}_{0}$ as $\kappa_{2}^{\mathrm{T}} \sim \mathcal{O}(100)$. This reflects the physical fact that the tidal term grows faster $\left(\sim 1 / r^{3}\right)$ at small separations than the nontidal one $\left(\sim 1 / r^{2}\right)$. Based on this picture, it is thus natural to interpret the NR data in terms of $\kappa_{2}^{\mathrm{T}}$ because the latter is the theoretically justified parameter that encodes the main effects of the EOS and masses on the dynamics.

Interestingly, the $\kappa_{2}^{\mathrm{T}}$ parameter approximately captures the collapse threshold and disk masses for nearly equal mass BNS $[12,35]$. On the one hand, this might be intuitive since $\kappa_{2}^{\mathrm{T}}$ contains information on the compactness of the binary. On the other hand it is not necessarily expected, given that the collapse is controlled by the maximum mass (pressure) supported by the EOS at densities much higher than those of the individual NSs. Thus, one should not expect the $\kappa_{2}^{\mathrm{T}}$ parameter to completely or accurately capture the strong-field dynamics; for this reason we defined the NR relations as quasiuniversal relations. For example, to capture the luminosity of binaries with mass ratios significantly different from unity, it is necessary to correct the leading-order post-Newtonian coefficient by a function of $\nu$ [12]. Similarly, in this paper we have introduced the parameter $\xi$ in Eq. (12) to better capture mass-ratio effects. The logic behind Eq. (12) is precisely to introduce a term that can account for the strong-field effect of $\hat{a}_{0}(\nu, u)$. However, for the reasons above, the $\xi$ parameter cannot 
properly describe quantities affected by significant tidal disruption. An extreme case is for example the disk mass in BH-NS binaries [108,109].

\section{APPENDIX B: BAYESIAN ANALYSIS WITH EOS INFERENCE}

Constraints on the matter EOS can be extracted from the GW signal by performing inference on a parametrized family of EOS [52,110-112]. Instead of sampling macroscopic EOS-related parameters, we can directly sample the function $p(\rho)$ that defines the EOS. Given this information, it is possible to infer the properties of each NS, such as tidal parameters and radii.

This method can be applied also with the complete model proposed in this work, as described in Sec. IV. In this case, it is possible to use Eq. (15) in combination with information from the EOS [instead of the tidal parameters and Eq. (16)], in order to infer whether the remnant undergoes a prompt collapse to a $\mathrm{BH}$. In particular, $M_{\max }^{\mathrm{TOV}}$ and the maximum NS compactness $C_{\max }$ would be calculated from the inferred EOS while $k_{\mathrm{thr}}$ can be estimated from the inferred $C_{\max }$ using NR fits [20,31]. This approach gives an alternative way to include prompt collapse in complete waveform models based on NRPM which we will further explore in future work.

\section{APPENDIX C: ROBUSTNESS OF NR POSTMERGER WAVEFORMS}

As discussed in the main text a main limitation in the construction of accurate postmerger models is the quality of NR postmerger waveforms. While the accuracy of inspiralmerger BNS waveforms has been studied in some detail and clear waveform convergence can be shown using high-order finite-differencing methods [79,80,113-115], the latter are less effective in postmerger simulations. Except for notable cases [29,58], the robustness of a postmerger waveform with grid resolution has not been studied in detail. We discuss here a resolution study of a long postmerger waveform.

Among the validation binaries, we simulated the evolution of the long-lived remnant employing a microphysical EOS SLy4 [116] starting from a binary system of individual NS masses of $1.30 M_{\odot}$ at different resolutions. These simulations span six orbits before merger and last for more than $100 \mathrm{~ms}$ after merger. Such integration times can be demanding in terms of computational time but NR codes allow stable evolutions at rather low grid resolution, e.g., [76,117-119]. Evolutions are performed with the WhiskyTHC code $[79,115,120,121]$ using a fifth-order monotonicity-preserving reconstruction within a standard second-order finite volume scheme [79]. Stars are covered with resolutions of $h=[0.415,0.246,0.185,0.135] \mathrm{km}$ in each direction, respectively, very low resolution (VLR), low resolution (LR), standard resolution (SR), and high

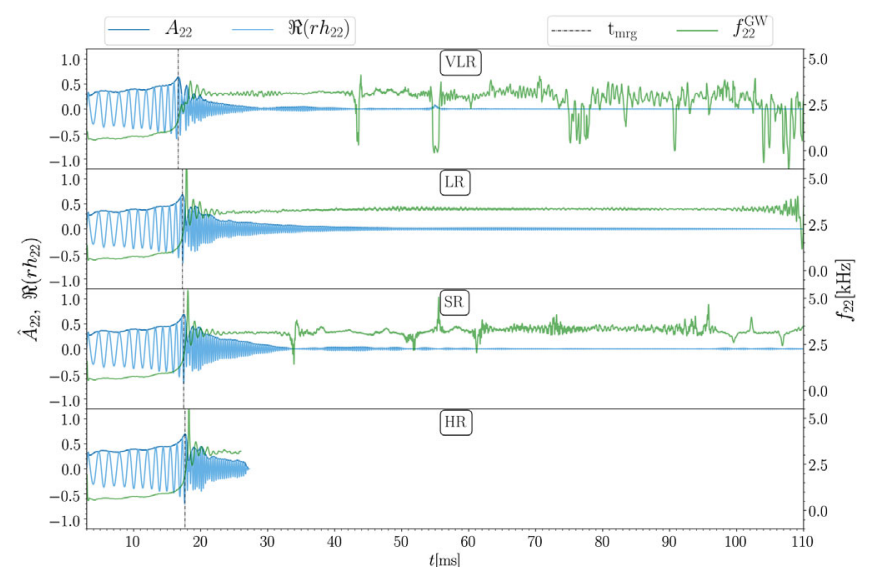

FIG. 13. Dependence of NR waveform on the grid resolution for the simulation SLy4 $M=(1.30+1.30) M_{\odot}$. VLR, LR, SR, HR stand respectively for maximal resolutions $h=$ $[0.415,0.246,0.185,0.136] \mathrm{km}$ in each direction.

resolution (HR), where SR is our standard for production runs [69] (but note that we performed also several HR simulations in past work). We use seven 2:1 refinement levels and a Courant-Friderich-Lewy factor of 0.075 for the time step.

The $(2,2)$ waveforms from runs at different resolution are shown in Fig. 13. The waveform's amplitude has a nonmonotonic behavior with increasing resolution. For example, the extrema in the time window $t \in(30,60) \mathrm{ms}$ are similar for VLR and SR but different from those of the LR data. The numerical high-frequency noise affecting the frequency decreases in magnitude the higher the resolution is, but it is mainly correlated to the amplitudes' minima. Hence, also the frequency noise is not converging with resolution at the considered resolutions. We checked the waveform phase convergence and found that the phase has a monotonic behavior with the grid resolution only until a few milliseconds after merger; the long-term data are not in the convergence regime at these resolutions.

Results at resolution VLR show the appearance of spurious frequencies at $f<f_{2}$ around $40 \mathrm{~ms}$; the latter are not present at higher resolutions. These frequencies have been erroneously interpreted as physical convective modes [118], which are instead not developed on these timescales even using a microphysical EOS. A careful inspection of the dynamics and multipolar waveform reveals instead physical spiral modes with $m=1$ geometry $[29,49,122,123]$. The GW frequency of the mode is $f_{1}=$ $f_{2} / 2$ and could be added to the NRPM model [49], but it corresponds to a weak GW emission [29].

We conclude that, to the best of the current knowledge, postmerger waveforms on timescales of $\sim 100 \mathrm{~ms}$ are well described in terms of the frequencies and amplitudes modeled by NRPM. The production of high-quality NR postmerger waveforms is an urgent goal. 
[1] B. P. Abbott et al. (Virgo and LIGO Scientific Collaborations), Phys. Rev. Lett. 119, 161101 (2017).

[2] B. P. Abbott et al. (LIGO Scientific and Virgo Collaborations), Phys. Rev. X 9, 011001 (2019).

[3] B. P. Abbott et al. (LIGO Scientific and Virgo Collaborations), Phys. Rev. X 9, 031040 (2019).

[4] S. Bernuzzi, A. Nagar, S. Balmelli, T. Dietrich, and M. Ujevic, Phys. Rev. Lett. 112, 201101 (2014).

[5] B. P. Abbott et al. (Virgo and LIGO Scientific Collaborations), Astrophys. J. 851, L16 (2017).

[6] B. P. Abbott et al. (LIGO Scientific and Virgo Collaborations), Astrophys. J. 875, 160 (2019).

[7] D. Lai and S. L. Shapiro, Astrophys. J. 442, 259 (1995).

[8] C. Cutler, Phys. Rev. D 66, 084025 (2002).

[9] A. Corsi and P. Mészáros, Astrophys. J. 702, 1171 (2009).

[10] S. Dall'Osso, B. Giacomazzo, R. Perna, and L. Stella, Astrophys. J. 798, 25 (2015).

[11] P. D. Lasky and K. Glampedakis, Mon. Not. R. Astron. Soc. 458, 1660 (2016).

[12] F. Zappa, S. Bernuzzi, D. Radice, A. Perego, and T. Dietrich, Phys. Rev. Lett. 120, 111101 (2018).

[13] J. A. Clark, A. Bauswein, N. Stergioulas, and D. Shoemaker, Classical Quantum Gravity 33, 085003 (2016).

[14] A. Torres-Rivas, K. Chatziioannou, A. Bauswein, and J. A. Clark, Phys. Rev. D 99, 044014 (2019).

[15] D. Martynov et al., Phys. Rev. D 99, 102004 (2019).

[16] S. Bernuzzi, T. Dietrich, and A. Nagar, Phys. Rev. Lett. 115, 091101 (2015).

[17] M. Shibata and K. Uryu, Phys. Rev. D 61, 064001 (2000).

[18] K. Kiuchi, Y. Sekiguchi, M. Shibata, and K. Taniguchi, Phys. Rev. D 80, 064037 (2009).

[19] K. Hotokezaka, K. Kyutoku, H. Okawa, M. Shibata, and K. Kiuchi, Phys. Rev. D 83, 124008 (2011).

[20] A. Bauswein, T. Baumgarte, and H. T. Janka, Phys. Rev. Lett. 111, 131101 (2013).

[21] T. Dietrich, M. Ujevic, W. Tichy, S. Bernuzzi, and B. Brügmann, Phys. Rev. D 95, 024029 (2017).

[22] T. Dietrich, S. Bernuzzi, M. Ujevic, and W. Tichy, Phys. Rev. D 95, 044045 (2017).

[23] M. Shibata and K. Uryu, Prog. Theor. Phys. 107, 265 (2002).

[24] N. Stergioulas, A. Bauswein, K. Zagkouris, and H.-T. Janka, Mon. Not. R. Astron. Soc. 418, 427 (2011).

[25] A. Bauswein and H.-T. Janka, Phys. Rev. Lett. 108, 011101 (2012).

[26] A. Bauswein, H. Janka, K. Hebeler, and A. Schwenk, Phys. Rev. D 86, 063001 (2012).

[27] K. Hotokezaka, K. Kiuchi, K. Kyutoku, T. Muranushi, Y.-I. Sekiguchi, M. Shibata, and K. Taniguchi, Phys. Rev. D 88, 044026 (2013).

[28] K. Takami, L. Rezzolla, and L. Baiotti, Phys. Rev. Lett. 113, 091104 (2014).

[29] D. Radice, S. Bernuzzi, and C. D. Ott, Phys. Rev. D 94, 064011 (2016).

[30] L. Lehner, S. L. Liebling, C. Palenzuela, O. L. Caballero, E. O'Connor, M. Anderson, and D. Neilsen, Classical Quantum Gravity 33, 184002 (2016).

[31] M. Agathos, F. Zappa, S. Bernuzzi, A. Perego, M. Breschi, and D. Radice, arXiv:1908.05442.
[32] B. Margalit and B. D. Metzger, Astrophys. J. 850, L19 (2017).

[33] A. Bauswein, O. Just, H.-T. Janka, and N. Stergioulas, Astrophys. J. 850, L34 (2017).

[34] M. Shibata, S. Fujibayashi, K. Hotokezaka, K. Kiuchi, K. Kyutoku, Y. Sekiguchi, and M. Tanaka, Phys. Rev. D 96, 123012 (2017).

[35] D. Radice, A. Perego, F. Zappa, and S. Bernuzzi, Astrophys. J. 852, L29 (2018).

[36] L. Rezzolla, E. R. Most, and L. R. Weih, Astrophys. J. 852, L25 (2018).

[37] Y.-W. Yu, L.-D. Liu, and Z.-G. Dai, Astrophys. J. 861, 114 (2018).

[38] S. Ai, H. Gao, Z.-G. Dai, X.-F. Wu, A. Li, B. Zhang, and M.-Z. Li, Astrophys. J. 860, 57 (2018).

[39] S.-Z. Li, L.-D. Liu, Y.-W. Yu, and B. Zhang, Astrophys. J. 861, L12 (2018).

[40] D. Lazzati, A. Deich, B. J. Morsony, and J. C. Workman, Mon. Not. R. Astron. Soc. 471, 1652 (2017).

[41] O. Bromberg, A. Tchekhovskoy, O. Gottlieb, E. Nakar, and T. Piran, Mon. Not. R. Astron. Soc. 475, 2971 (2018).

[42] K. Chatziioannou, J. A. Clark, A. Bauswein, M. Millhouse, T. B. Littenberg, and N. Cornish, Phys. Rev. D 96, 124035 (2017).

[43] C. Helstrom, Statistical theory of signal detection, International Series of Monographs on Electronics and Instrumentation (Pergamon Press, New York, 1968).

[44] P. J. Easter, P. D. Lasky, A. R. Casey, L. Rezzolla, and K. Takami, Phys. Rev. D 100, 043005 (2019).

[45] A. Bauswein, N. Stergioulas, and H.-T. Janka, Eur. Phys. J. A 52, 56 (2016).

[46] S. Bose, K. Chakravarti, L. Rezzolla, B. S. Sathyaprakash, and K. Takami, Phys. Rev. Lett. 120, 031102 (2018).

[47] K. W. Tsang, T. Dietrich, and C. Van Den Broeck, Phys. Rev. D 100, 044047 (2019).

[48] A. Bauswein, N. Stergioulas, and H.-T. Janka, Phys. Rev. D 90, 023002 (2014).

[49] L. Lehner, S. L. Liebling, C. Palenzuela, and P. M. Motl, Phys. Rev. D 94, 043003 (2016).

[50] L. Rezzolla and K. Takami, Phys. Rev. D 93, 124051 (2016).

[51] K. Kiuchi, K. Kyohei, K. Kyutoku, Y. Sekiguchi, and M. Shibata, arXiv:1907.03790.

[52] B. P. Abbott et al. (LIGO Scientific, Virgo Collaborations), Phys. Rev. Lett. 121, 161101 (2018).

[53] A. Perego, S. Bernuzzi, and D. Radice, Eur. Phys. J. A 55, 124 (2019).

[54] J. Antoniadis, P. C. Freire, N. Wex, T. M. Tauris, R. S. Lynch et al., Science 340, 1233232 (2013).

[55] H. T. Cromartie et al., arXiv:1904.06759.

[56] A. W. Steiner, J. M. Lattimer, and E. F. Brown, Eur. Phys. J. A 52, 18 (2016).

[57] Y. Sekiguchi, K. Kiuchi, K. Kyutoku, and M. Shibata, Phys. Rev. Lett. 107, 211101 (2011).

[58] D. Radice, S. Bernuzzi, W. Del Pozzo, L. F. Roberts, and C. D. Ott, Astrophys. J. 842, L10 (2017).

[59] A. Bauswein, N.-U.F. Bastian, D. B. Blaschke, K. Chatziioannou, J. A. Clark, T. Fischer, and M. Oertel, Phys. Rev. Lett. 122, 061102 (2019). 
[60] E. R. Most, L. J. Papenfort, V. Dexheimer, M. Hanauske, S. Schramm, H. Stöcker, and L. Rezzolla, Phys. Rev. Lett. 122, 061101 (2019).

[61] T. Dietrich, D. Radice, S. Bernuzzi, F. Zappa, A. Perego, B. Brügmann, S. V. Chaurasia, R. Dudi, W. Tichy, and M. Ujevic, Classical Quantum Gravity 35, 24LT01 (2018).

[62] T. Dietrich et al., Phys. Rev. D 99, 024029 (2019).

[63] E. E. Flanagan and T. Hinderer, Phys. Rev. D 77, 021502 (2008).

[64] T. Damour and A. Nagar, Phys. Rev. D 81, 084016 (2010).

[65] T. Damour, in Gravitational Radiation, edited by $\mathrm{N}$. Deruelle and T. Piran (North-Holland, Amsterdam, 1983), pp. 59-144.

[66] T. Hinderer, Astrophys. J. 677, 1216 (2008).

[67] T. Damour and A. Nagar, Phys. Rev. D 80, 084035 (2009).

[68] T. Binnington and E. Poisson, Phys. Rev. D 80, 084018 (2009).

[69] D. Radice, A. Perego, K. Hotokezaka, S. A. Fromm, S. Bernuzzi, and L.F. Roberts, Astrophys. J. 869, 130 (2018).

[70] H. Dimmelmeier, N. Stergioulas, and J. A. Font, Mon. Not. R. Astron. Soc. 368, 1609 (2006).

[71] A. Passamonti, N. Stergioulas, and A. Nagar, Phys. Rev. D 75, 084038 (2007).

[72] L. Baiotti, S. Bernuzzi, G. Corvino, R. De Pietri, and A. Nagar, Phys. Rev. D 79, 024002 (2009).

[73] K. Takami, L. Rezzolla, and L. Baiotti, Phys. Rev. D 91, 064001 (2015).

[74] A. Bauswein and N. Stergioulas, Phys. Rev. D 91, 124056 (2015).

[75] S. Bernuzzi, D. Radice, C. D. Ott, L. F. Roberts, P. Moesta, and F. Galeazzi, Phys. Rev. D 94, 024023 (2016).

[76] R. Ciolfi, W. Kastaun, J. V. Kalinani, and B. Giacomazzo, Phys. Rev. D 100, 023005 (2019).

[77] F. Zappa, Master's thesis, Parma University, 2018.

[78] S. Bernuzzi, T. Dietrich, W. Tichy, and B. Brügmann, Phys. Rev. D 89, 104021 (2014).

[79] D. Radice, L. Rezzolla, and F. Galeazzi, Classical Quantum Gravity 31, 075012 (2014).

[80] S. Bernuzzi and T. Dietrich, Phys. Rev. D 94, 064062 (2016).

[81] S. Banik, M. Hempel, and D. Bandyopadhyay, Astrophys. J. Suppl. Ser. 214, 22 (2014).

[82] C. Cutler and E. E. Flanagan, Phys. Rev. D 49, 2658 (1994).

[83] J. Aasi et al. (LIGO Scientific Collaboration), Classical Quantum Gravity 32, 115012 (2015).

[84] B. P. Abbott et al. (VIRGO, KAGRA, LIGO Scientific Collaborations), Living Rev. Relativity 21, 3 (2018).

[85] G. M. Harry (LIGO Scientific Collaboration), Classical Quantum Gravity 27, 084006 (2010).

[86] S. Bernuzzi, A. Nagar, T. Dietrich, and T. Damour, Phys. Rev. Lett. 114, 161103 (2015).

[87] T. Dietrich, S. Bernuzzi, M. Ujevic, and B. Brügmann, Phys. Rev. D 91, 124041 (2015).

[88] L. Lindblom, B. J. Owen, and D. A. Brown, Phys. Rev. D 78, 124020 (2008).

[89] L. Lindblom, Phys. Rev. D 80, 064019 (2009).

[90] A. Nagar et al., Phys. Rev. D 98, 104052 (2018).
[91] M. Punturo, M. Abernathy, F. Acernese, B. Allen, N. Andersson et al., Classical Quantum Gravity 27, 194002 (2010).

[92] S. Hild et al., Classical Quantum Gravity 28, 094013 (2011).

[93] S. Akcay, S. Bernuzzi, F. Messina, A. Nagar, N. Ortiz, and P. Rettegno, Phys. Rev. D 99, 044051 (2019).

[94] F. Acernese et al. (Virgo Collaboration), Classical Quantum Gravity 32, 024001 (2015).

[95] J. Skilling, Bayesian Anal. 1, 833 (2006).

[96] J. Veitch and A. Vecchio, Phys. Rev. D 81, 062003 (2010).

[97] J. Veitch et al., Phys. Rev. D 91, 042003 (2015).

[98] LIGO Scientific Collaboration, LIGO Algorithm Library -LALSuite, free software (GPL) (2018).

[99] B. D. Lackey, S. Bernuzzi, C. R. Galley, J. Meidam, and C. Van Den Broeck, Phys. Rev. D 95, 104036 (2017).

[100] B. D. Lackey, K. Kyutoku, M. Shibata, P. R. Brady, and J. L. Friedman, Phys. Rev. D 85, 044061 (2012).

[101] J. S. Read, L. Baiotti, J. D. E. Creighton, J. L. Friedman, B. Giacomazzo, K. Kyutoku, C. Markakis, L. Rezzolla, M. Shibata, and K. Taniguchi, Phys. Rev. D 88, 044042 (2013).

[102] A. Ghosh et al., Phys. Rev. D 94, 021101 (2016).

[103] B. P. Abbott et al. (LIGO Scientific and Virgo Collaborations), Phys. Rev. Lett. 116, 221101 (2016); 121, 129902 (E) (2018).

[104] B. P. Abbott et al. (LIGO Scientific and Virgo Collaborations), arXiv:1903.04467.

[105] M. Breschi, R. O’Shaughnessy, J. Lange, and O. Birnholtz, arXiv:1903.05982.

[106] K. Kiuchi, K. Kawaguchi, K. Kyutoku, Y. Sekiguchi, M. Shibata, and K. Taniguchi, Phys. Rev. D 96, 084060 (2017).

[107] K. Kawaguchi, K. Kiuchi, K. Kyutoku, Y. Sekiguchi, M. Shibata, and K. Taniguchi, Phys. Rev. D 97, 044044 (2018).

[108] F. Foucart, T. Hinderer, and S. Nissanke, Phys. Rev. D 98, 081501 (2018).

[109] F. Zappa, S. Bernuzzi, F. Pannarale, M. Mapelli, and N. Giacobbo, Phys. Rev. Lett. 123, 041102 (2019).

[110] L. Lindblom, Phys. Rev. D 82, 103011 (2010).

[111] B. D. Lackey and L. Wade, Phys. Rev. D 91, 043002 (2015).

[112] M. F. Carney, L. E. Wade, and B. S. Irwin, Phys. Rev. D 98, 063004 (2018).

[113] S. Bernuzzi, M. Thierfelder, and B. Brügmann, Phys. Rev. D 85, 104030 (2012).

[114] S. Bernuzzi, A. Nagar, M. Thierfelder, and B. Brügmann, Phys. Rev. D 86, 044030 (2012).

[115] D. Radice, L. Rezzolla, and F. Galeazzi, Mon. Not. R. Astron. Soc. 437, L46 (2014).

[116] A. S. Schneider, L. F. Roberts, and C. D. Ott, Phys. Rev. C 96, 065802 (2017).

[117] N. Andersson, J. Baker, K. Belczynski, S. Bernuzzi, E. Berti et al., Classical Quantum Gravity 30, 193002 (2013).

[118] R. De Pietri, A. Feo, J. A. Font, F. Löffler, F. Maione, M. Pasquali, and N. Stergioulas, Phys. Rev. Lett. 120, 221101 (2018).

[119] V. Nedora, S. Bernuzzi, D. Radice, A. Perego, A. Endrizzi, and N. Ortiz, arXiv:1907.04872. 
[120] D. Radice and L. Rezzolla, Astron. Astrophys. 547, A26 (2012).

[121] D. Radice, A. Perego, S. Bernuzzi, and B. Zhang, Mon. Not. R. Astron. Soc. 481, 3670 (2018).
[122] V. Paschalidis, W. E. East, F. Pretorius, and S. L. Shapiro, Phys. Rev. D 92, 121502 (2015).

[123] W. E. East, V. Paschalidis, and F. Pretorius, Classical Quantum Gravity 33, 244004 (2016). 